\title{
CONTRACT ADJUSTMENT UNDER UNCERTAINTY
}

\author{
HELGE HOLDEN \\ LARS HOLDEN \\ STEINAR HOLDEN
}

CESIFO WORKING PAPER NO. 1472

CATEGORY 10: EMPIRICAL AND THEORETICAL METHODS

MAY 2005

An electronic version of the paper may be downloaded

- from the SSRN website:

www.SSRN.com

- from the CESifo website:

www.CESifo.de 


\title{
CONTRACT ADJUSTMENT UNDER UNCERTAINTY
}

\begin{abstract}
Consider a contract over trade in continuous time between two players, according to which one player makes a payment to the other, in exchange for an exogenous service. At each point in time, either player may unilaterally require an adjustment of the contract payment, involving adjustment costs for both players. Players' payoffs from trade under the contract, as well as from trade under an adjusted contract, are exogenous and stochastic. We consider players' choice of whether and when to adjust the contract payment. It is argued that the optimal strategy for each player is to adjust the contract whenever the contract payment relative to the outcome of an adjustment passes a certain threshold, depending among other things of the adjustment costs. There is strategic substitutability in the choice of thresholds, so that if one player becomes more aggressive by choosing a threshold closer to unity, the other player becomes more passive. If players may invest in order to reduce the adjustment costs, there will be over-investment compared to the welfare maximizing levels.
\end{abstract}

JEL Code: C72, C73, C78, E31.

Helge Holden

Department of Mathematical Sciences

Norwegian University of Science and

Technology

7491 Trondheim

Norway

holden@math.ntnu.no
Lars Holden

Norwegian Computing Center

P.O. Box 114 Blindern

0314 Oslo

Norway

lars.holden@nr.no

Steinar Holden

Department of Economics

University of Oslo

P.O. Box 1095 Blindern

0317 Oslo

Norway

steinar.holden@econ.uio.no 


\section{INTRODUCTION}

In most economies, a large part of the transactions take place within long-term relationships. Generally, players' payoffs from these transactions depend on the rules or norms that govern the relationship. For example, the payoffs to the parties in an employment relationship depend on the labor contract. The payoffs to two partners in a partnership depend on how they share the benefits and work within their relationship. The rules or norms in a relationship may be written down in a formal contract, or they may just be given by a mutual understanding of "how things are done". In either case, the rules and norms are often constant over long time, until one player demands that they are changed. In this paper we analyze the decision of whether and when to demand that the rules are changed. For concreteness, we choose one specific type of transactions and rules, where one player undertakes an exogenous service for the other, and where the remuneration for the service is given in a contract. Trade takes place in continuous time, and players' flow payoffs depend on the prevailing contract.

Typically, a demand for adjustment of the contract may be motivated by changes in the "inside" or the "outside" option. First, players' payoff from trade under the contract may change to one player's disadvantage, causing this player to require an adjustment of the contract. We capture this effect by assuming, realistically, that the contract payment is set in nominal terms, so that the real value of the contract payment depends on the stochastic aggregate price level. Second, outside alternatives may change, which we capture by assuming that the outcome of an adjustment of the contract is given by an exogenous stochastic process, known to both parties at the time when an adjustment is demanded.

Formally, we consider a two-player differential game. There is a contract, according to which player B (the buyer) makes a fixed nominal payment $V$ per unit of time to player A (the seller), as a remuneration for some exogenous and unspecified service. The real value of the fixed payment, $R$, depends on the aggregate price level $Q$, where $R=V / Q$. At each point in time, either player may unilaterally induce an adjustment of the contract payment to a new real value $Z$, which we shall refer to as the real adjustment outcome (implying a new nominal value $V=Z Q$ ). Adjusting the contract payment carries an exogenous fee to both players. The aggregate price level $Q$ and the real adjustment outcome $Z$ are exogenous stochastic processes. We consider players' decision of when to adjust the payment given in the contract, allowing for an unlimited number of adjustments over an infinite horizon.

The model we consider is simple: There are only two players, and their only choice variable is at each point in time whether to demand an adjustment of the contract. The result of a possible adjustment is known to both players. Yet the decision problem facing the players is very complex. When deciding whether to require an adjustment of the contract, a player must weigh the gain from a possible improvement in contract terms against the costs of adjustment. However, the player must also take into consideration that an adjustment now, making the contract terms more favorable to himself, will make the contract less favorable to the opponent. This may cause the opponent to require an adjustment at an earlier point in time than he otherwise would have done, involving both adjustment costs and less favorable contract terms for the first player.

In principle, strategies may depend on anything that has happened in the history of the game, and thus be immensely complicated. To keep the analysis tractable, we follow the tradition of the differential games literature (see Isaacs [17] and Dockner, Jørgensen, Van Long, and Sorger [10]) of restricting attention to Markov strategies, i.e., strategies where actions are allowed to depend on past history through the current value of the state variables only.

We show that the optimal strategies of the players are given by critical thresholds for the ratio between the real contract payment, $R$, and the real adjustment outcome, $Z$. After each adjustment, $R$ is set equal to $Z$, implying that the ratio $R / Z=1$. In equilibrium, player A will demand an adjustment whenever $R / Z$ is below player A's threshold, irrespective of whether this is caused by high inflation eroding the real value of the contract payment, or by an increase in the real adjustment outcome. Conversely, player $\mathrm{B}$ will require an adjustment whenever $R / Z$ is above player B's threshold. 
The thresholds depend, among other things, on the costs of adjustment and the expected drift and volatility of the stochastic processes. As expected, we show that the higher the costs of adjustment for a player, the more passive is the player, i.e., the further the critical threshold is from unity. More volatility in the stochastic processes widens the interval between the thresholds, yet adjustments occur more frequently.

Our key result is that there is strategic substitutability in the choice of threshold values, so that if one player becomes more aggressive (i.e., setting a threshold closer to unity), the opponent will become more modest (i.e., setting a threshold further from unity). The intuition for this result is that if a player becomes more aggressive, the expected time until this player requires an adjustment is reduced. Thus, the expected duration of a change in contract terms induced by the opponent is reduced, which makes it less attractive for the opponent to require an adjustment.

One implication of strategic substitutability is that asymmetries between the players may be exacerbated. For example, if adjustment becomes less costly for one of the players, making this player more aggressive, the strategic effect will make the opponent more passive. As the opponent becomes more passive, the first player becomes even more aggressive, exacerbating the direct effect of reduced adjustment costs. Numerical examples suggest that the strategic effect in some cases may be substantial.

The paper is related to several different strands of literature. As noted above, the model is formally a type of differential game. Economically, the paper is closer related to the considerable literature which studies the optimal choice of nominal prices (or wages) under a stochastic evolution of money or aggregate prices (see, e.g., Sheshinski and Weiss [23], Danziger [7], Caplin and Spulber [6], and Caplin and Leahy [5]). As in our paper, adjustment of the nominal price is costly. As in our model, optimal behavior under state-dependent price setting is typically characterized by threshold strategies, often termed $(s, S)$ strategies, where the prevailing price is changed if it is sufficiently far from the optimal new price, so that the gain from adjustment covers the adjustment costs. Compared to this literature, we simplify by taking the adjustment outcome as exogenous, focusing solely on the timing decision. On the other hand, by considering a two-player game, we introduce a strategic dimension. Thus, when deciding whether to adjust the contract payment now, a player must take into consideration that this may cause a subsequent adjustment by the opponent, at an earlier stage than he otherwise would have done, inflicting additional costs on both players.

Our paper is also related to the literature on the duration of labor contracts, see Danziger [9] for a recent contribution with reference to previous literature. Most of this literature is concerned about the duration of fixed-length (or time-dependent) wage-contracts, empirically, or as seen from the point of optimality. In contrast, the main focus of the present paper is on the adjustment decision in a state-dependent setting.

The model we consider is very similar to the model studied in Andersen and Christensen [3]. As in our model, Andersen and Christensen consider bilateral trade in continuous time according to a given contract, and players' only decision is at each point in time whether to demand an adjustment of the contract (which Andersen and Christensen refer to as a renegotiation of the contract). The outcome of the renegotiation is assumed to be given by a geometric Brownian motion. However, an important limitation of Andersen and Christensen [3] is that it only allows for one contract renegotiation, implying that if one player has required a renegotiation, this option is no longer open to the other player. Thus, Andersen and Christensen find strategic complementarity in players' contract renegotiation decisions, i.e., that the more reluctant one player is to demand a renegotiation, the more reluctant the opponent will be, in contrast to our finding of strategic substitutability.

In Andersen and Christensen [2] the model is extended to a large but finite number of contract renewals, and the model is solved by use of backward induction from the last possible contract renewal. However, it is not stated whether the strategic complementarity also holds in this case.

Our analysis is relevant for bilateral trade under a contract, e.g., a labor contract, a tenancy agreement, or a delivery contract. Such contracts often specify an expiration date, seemingly making the decision of when to require an adjustment less relevant. However, in spite of an expiration date, such contracts are often renegotiated before the expiration date, or extended 
considerably beyond the date, making the decision of when to adjust the contract important. In some contracts, there is an explicit renegotiation clause, allowing either of the parties to unilaterally request a renegotiation. If the contract does not include a renegotiation clause, a player may nevertheless enforce a renegotiation by credibly threatening to disrupt trade, if the opponent prefers a renegotiation to a disruption of trade. Such threats may be possible if it is not verifiable for the court which party violates the contract (MacLeod and Malcomson [19] and Hart and Moore [13], or if the courts will not enforce any penalty provisions (Grout [11]). Thus, in practice, even contracts without a renegotiation clause are occasionally renegotiated or terminated if a sufficiently large shock takes place.

As to extension beyond the expiration date, many contracts have legal effects even after the expiration date. In many countries, parties to an employment contract are legally bound by the terms of the contract, even after the expiration date, unless both parties have agreed on a new contract, or one party has terminated the relationship, see Malcomson [20] and Holden [14]. As an illustration that contract extension really happens, consider the sample of Israeli labor contracts studied by Danziger [8]: 86 percent of all new contracts were signed after the expiration of the previous contract, with average delay 213 days. Again, the decision of when to adjust the contract is relevant.

We believe that our analysis is also relevant for other applications. For example, the relationship between two countries may be governed by various types of agreements, like trade agreements or environmental agreements. A country may want to renegotiate such agreements if it believes that this will lead to more beneficial terms. If one country is aggressive, in the sense of being eager to exploit any possibility of improving the terms of trade, how will this affect the strategy of the other? Another example is two partners in a business partnership, where the partners' respective payoffs depend on how they share benefits and costs. Here, too, a partner may request an adjustment of the shares if relevant circumstances change, e.g., a change in the partners' real payoffs from the partnership, or in the payoffs that can be obtained in alternative relationships.

The remainder of the paper is organized as follows. The basic model is described in Section 2. Here we show that there exists no strategy that is better than a threshold strategy, specifying an adjustment of the contract whenever the ratio of the real contract payment to the real adjustment outcome passes a specific threshold. Section 3 analyzes the case when both players use threshold strategies. In Section 4, we impose the assumption that the stochastic environment is continuous, and we prove the existence of a Nash equilibrium. In Section 5, we again allow for discontinuities in the stochastic environment, and show that in this case equilibrium may involve randomization. In Section 6, we extend the basic model by allowing for a stage prior to the basic model, where players may invest in reducing the adjustment cost, and we consider the efficiency of this investment decision. Section 7 summarizes some of the main results. An approximate formula for the equilibrium is given in Appendix A. Proofs are provided in Appendix B. The case where only one player is allowed to adjust the contract has been analyzed in detail in a working paper. For brevity reasons these results are not included in the present paper.

\section{THE MODEL}

We consider a two-player differential game. There is a contract, according to which player B makes a fixed nominal payment $V\left(t_{i}\right)$ per unit of time to player $\mathrm{A}$, as a remuneration for some exogenous and unspecified service. The time when the payment is set, is denoted $t_{i}$. At each point in time, either of the players may unilaterally adjust the nominal payment, inducing an adjustment cost on both players.

The real value of the contract payment at time $t, R(t, \omega)$ is found by deflating the nominal contract payment by the aggregate price level $Q(t, \omega)$ at time $t$, i.e., $R(t, \omega)=V\left(t_{i}\right) / Q(t, \omega)$. The parameter $\omega$ denotes that $Q(t, \omega)$ and thus also $R(t, \omega)$ are stochastic. Players' flow payoffs are constant elasticity functions of the real contract payment, so that $R^{\eta_{A}}$ (i.e., $R$ raised to the power $\left.\eta_{A}\right)$ and $R^{\eta_{B}}$ are the flow payoffs of player $\mathrm{A}$ and $\mathrm{B}$, respectively, and where the respective rates 
of risk aversion are $\eta_{A}>0$ and $\eta_{B}<0$ (implying that player A gains and player B loses from an increase in $R)^{1}{ }^{1}$

If a player demands an adjustment of the contract at time $t$, the real value of the new contract is set equal to the real adjustment outcome $Z(t, \omega)$, which is also an exogenous stochastic process. The new nominal contract is thus $V(t, \omega)=Z(t, \omega) Q(t, \omega)$. Adjustment of the contract involves a fee that is proportional to the real adjustment outcome; specifically, the adjustment fee is $\tau_{\nu} Z^{\eta_{\nu}}$, where $\tau_{\nu}(\nu=A$ or $B)$ is assumed to be strictly positive, deterministic and independent of which player is initiating the adjustment. ${ }^{2}$ (This assumption may be generalized, see observation II in Section 4.)

Note that we do not consider the possibility that players care explicitly about the actions and intentions of the other player. Thus, we neglect that a player may care about an adjustment per se, viewing it as unfair or unwarranted; it is only the real contract payment and the adjustment costs that enter the payoff functions.

The overall objective function of the players is the discounted sum of flow payoffs

$$
U_{\nu}\left(t_{1}, \ldots, \omega\right)=\int_{0}^{\infty} R^{\eta_{\nu}}(s, \omega) \exp (-\beta s) d s-\tau_{\nu} \sum_{j=0}^{\infty} Z^{\eta_{\nu}}\left(t_{j+1}, \omega\right) \exp \left(-\beta t_{j+1}\right)
$$

where the discount rate $\beta>0$ and $t_{j}$ the times of contract adjustment. To avoid unimportant additional constants, we normalize by setting $R(0, \omega)=Z(0, \omega)=1$, and $t_{0}=0$. As noted above, the players choose when to adjust the contract, so as to maximize their objective function. At each time $t$, the contemporaneous values $Z(t, \omega)$ and $Q(t, \omega)$ are known to the players, but the future values $Z(s, \omega)$ and $Q(s, \omega)$ for $s>t$ are unknown.

One may argue that when the outcome of the adjustment is known to the players in advance, the adjustment costs, which reflect time and uncertainty associated with agreeing upon a new contract, should be negligible. However, it is straightforward, but cumbersome, to extend the model so that players at time $t$ only know the expected outcome of the adjustment at time $t$, and where the actual adjustment outcome at time $t$ is stochastic. In this extension, the expected outcome might either be a function of the previous adjustment outcome, i.e., for $t>t_{i}$, the expected outcome in real terms is described by $E\left\{Z(t) \mid Z\left(t_{i}, \omega\right)\right\}=\exp \left(c\left(t-t_{i}\right)\right) Z\left(t_{i}, \omega\right)$ for a constant $c$, or the expected outcome might be a stochastic process similar to $Z$ and $Q$ in the present model. Under both alternatives, the qualitative results would be unaffected.

It might seem plausible that the real adjustment outcome $Z$ would depend on the players' adjustment costs, or the preference function, so that a player with lower adjustment costs, or a less risk averse player, has a stronger position in the adjustment process, and thus is able to obtain a better outcome. The fact that there is an existing contract may also have profound impact on the renegotiation outcome, see MacLeod and Malcomson [19] and Holden [16]. However, making the adjustment or renegotiation outcome endogenous would complicate the analysis considerably, making key aspects less transparent. Thus, we neglect such aspects and treat $Z$ as exogenous.

To ensure a high degree of generality, we assume that the real adjustment outcome and the aggregate price level are given by the exponential of a Lévy process. Thus we assume that $Z(s, \omega)=$ $\exp (F(s, \omega))$ and $Q(s, \omega)=\exp (G(s, \omega))$ where $F, G$ are Lévy processes. Lévy processes include geometric Brownian motion, jump processes that follow a Poisson distribution and many other stochastic processes that are, e.g., asymmetric or have heavier tails. For the benefit of the reader we recall the definition of a Lévy process (see, e.g., Sato [22]).

\footnotetext{
${ }^{1}$ The payoff functions can be derived from a collective bargaining framework, where workers' payoff is assumed to be an increasing and isoelastic function of the real wage, $R$. For a price setting firm, with a constant elasticity production function, labor as the only input, and constant elasticity of product demand, it is straightforward to show that real profits will be a decreasing isoelastic function of the real wage. We further allow the firm's payoff to be an isoelastic function of real profits. Note, however, that as $\eta_{A}>0$ and $\eta_{B}<0$, the model is not symmetric. Yet by use of the same method, it can be shown that the analysis and results would be qualitatively the same in a symmetric model where the flow payoff of player $\mathrm{B}$ is $-R^{\eta_{B}}$, and $\eta_{B}=\eta_{A}>0$.

${ }^{2}$ Proportional adjustment fees, adjusted for the constant elasticity $\eta_{\nu}$, yield tractable solutions. In a labor contract, adjustment costs may reflect time spent on bargaining, and the real contract payment (i.e., the real wage) seems an appropriate measure of the costs of time.
} 
Definition 2.1 (Lévy process). A stochastic process $X_{t}$ is a Lévy process provided the following conditions hold:

(i) For any $n$ and any $0 \leq t_{0}<\cdots<t_{n}$ the random variables $X_{t_{0}}, X_{t_{1}}-X_{t_{0}}, \ldots, X_{t_{n}}-X_{t_{n-1}}$ are independent.

(ii) $X_{0}=0$ almost surely.

(iii) The distribution of $X_{s+t}-X_{s}$ is independent of $s$.

(iv) The process is stochastically continuous, i.e., $\lim _{t \downarrow 0} \operatorname{Prob}\left(\left|X_{t}\right|>\epsilon\right)=0$ for all $\epsilon>0$.

(v) The process is right-continuous with left limits.

For Lévy processes we have the Lévy-Khintchine formula for the characteristic function of $X_{t}$ (see, e.g., Sato [22])

$$
E\left\{\exp \left(i \lambda X_{t}\right)\right\}=\exp \left(t\left(i \alpha \lambda-\frac{1}{2} \lambda^{2} a^{2}+\int_{-\infty}^{\infty}\left(e^{i \lambda x}-1-i \lambda x \chi_{\{|x| \leq 1\}}(x)\right) d \sigma(x)\right)\right),
$$

where $d \sigma$ is a $\sigma$-finite measure, denoted the Lévy-measure, with $\int_{-\infty}^{\infty} \min \left(|x|^{2}, 1\right) d \sigma(x)<\infty$ and $\sigma(\{0\})=0$. The process is uniquely defined by the quantities $(\alpha, a, d \sigma)$. The measure $\sigma$ describes the size and intensity of the jumps in the process. The process is Gaussian if and only if $\sigma=0$, and in that case, $\alpha$ denotes the drift and $a$ the volatility. If $\sigma$ satisfies

$$
\int_{1}^{\infty} e^{\eta x} d \sigma(x)<\infty
$$

we may conclude that

$$
E\left\{\exp \left(\eta X_{t}\right)\right\}=\exp \left(t\left(\alpha \eta+\frac{1}{2} \eta^{2} a^{2}+\int_{-\infty}^{\infty}\left(e^{\eta x}-1-\eta x \chi_{\{|x| \leq 1\}}(x)\right) d \sigma(x)\right)\right)
$$

holds and is finite.

To ensure that the objective functions are finite, it is necessary to bound $Z$ and $Q$ relative to the discount rate $\beta$. This requires two additional assumptions. First, we assume that the volatility of the non-gaussian part is bounded, by assuming that the Lévy-measures satisfy

$$
\int_{1}^{\infty} e^{\eta_{h} x} d \sigma_{h}(x)<\infty, \quad h=z, q
$$

for some $\eta_{h}$. We may then define the drift in the processes for the real adjustment outcome $Z$ and the aggregate price level $Q$ by

$$
\mu_{\nu, h}=\alpha_{h} \eta_{\nu}+\frac{1}{2} \eta_{\nu}^{2} a_{h}^{2}+\int_{-\infty}^{\infty}\left(e^{\eta_{\nu} x}-1-\eta_{\nu} x \chi_{\{|x| \leq 1\}}(x)\right) d \sigma_{h}(x), \quad \eta_{\nu} \leq \eta_{h},
$$

for $\nu=A, B$ and $h=z, q$. We have that $E\left\{Z^{\eta_{\nu}}(t, \omega)\right\}=\exp \left(t \mu_{\nu, z}\right)$ and $E\left\{Q^{\eta_{\nu}}(t, \omega)\right\}=\exp \left(t \mu_{\nu, q}\right)$. For example, $\mu_{A, z}$ is the expected rate of increase in the real adjustment payment, adjusted for the relative rate of risk aversion of player $\mathrm{A}, \eta_{A}$. The second assumption is that the drift parameters $\mu_{\nu, h}$ must be bounded by the discount rate $\beta$.

Definition 2.2 (Property $\mathcal{F})$. We say that the stochastic contract model has property $\mathcal{F}$ if the following properties hold:

(i) The real adjustment outcome $Z(s, \omega)=\exp (F(s, \omega))$ and $Q(s, \omega)=\exp (G(s, \omega))$ where $F$ and $G$ are Lévy processes given by $\left(\alpha_{z}, a_{z}, d \sigma_{z}\right)$ and $\left(\alpha_{q}, a_{q}, d \sigma_{q}\right)$, respectively.

(ii) There exists $\eta_{h}$ such that

$$
\int_{1}^{\infty} e^{\eta_{h} x} d \sigma_{h}(x)<\infty, \quad h=z, q
$$

and assume that $\eta_{\nu} \leq \eta_{h}$ for $\nu=A, B$ and $h=z, q$.

(iii) We have that

$$
\mu_{\nu, h}<\beta, \quad \nu=A, B, h=z, q .
$$


Note that by assuming that payoff functions exhibit constant elasticity in the real contract payment $R$, and that the stochastic processes are given by the exponential of Lévy processes, we ensure that the situation is the same after each adjustment, subject to a constant $Z(t, \omega)$. This property is crucial for the analysis, as it implies that the same strategies are optimal after each adjustment.

The strategy of a player is defined as a description of the criteria that apply for when the player will require an adjustment of the contract. In principle, strategies may depend on anything that has happened in the history of the game. However, we will follow the tradition of the differential games literature and restrict attention to Markov strategies, where the players' choice of action only depend the state of the game. Thus, players may condition their play on the real contract payment $R$, the real adjustment outcome $Z$, or any combination of these variables. We do not allow players to condition their play on the opponent's play, except for any effect via the state variables $R$ and $Z$. For example, we do not consider strategies where players punish a rapid adjustment by the opponent by another adjustment, inflicting further adjustment costs on both players.

The theorem below states that if one of the players uses a Markov strategy, there exists no strategy for the other player that gives higher expected value of the objective function than having a critical threshold for the ratio $R / Z$, i.e adjusting the contract whenever $R / Z$ is equal to or passes a certain threshold value. Other variables like $R$ or $Z$ separately, calendar time or the time duration since the previous adjustment, need not be used in the strategy.

Theorem 2.3. Assume the stochastic contract model satisfies property $\mathcal{F}$. Assume that one player uses a Markov strategy. Then there exists no strategy for the other player that gives higher expected payoff than the payoff that can be obtained with a threshold strategy based on the ratio $R / Z$.

Let $r_{B}$ and $r_{A}$ denote the critical thresholds, where $r_{A}<1<r_{B}$, as player A requires adjustment if the real contract payment is low $\left(R / Z \leq r_{A}\right)$, while player $\mathrm{B}$ requires adjustment if the real contract payment is high $\left(R / Z \geq r_{B}\right)$. Note that Theorem 2.3 does not imply that there exists a pair $\left(r_{A}, r_{B}\right)$ where $r_{A}$ is the optimal response to $r_{B}$ and $r_{B}$ is the optimal response $r_{A}$; in Section 5, a counterexample is given.

\section{THE MODEL WHEN BOTH PLAYERS USE THRESHOLDS STRATEGIES}

In the previous section it was proved that if one player uses a Markov strategy, the opponent has no strategy that is better than what can be achieved by a threshold strategy for the ratio $R / Z$. Thus, in this and the following sections we assume that both players use such threshold strategies. In this section we derive formulas for the expected objective functions and their derivatives. These formulas can be computed numerically, and possibly also analytically, for specific stochastic processes. To illustrate the model, figure 1 shows a realization of the real contract payment $R$ when both players use threshold strategies.

Define the expected discounted sum of flow payoffs ${ }^{3}$

$$
u_{\nu}\left(r_{A}, r_{B}\right)=E\left\{U_{\nu}\left(r_{A}, r_{B}\right)\right\}
$$

where $U_{\nu}\left(r_{A}, r_{B}, \omega\right)$, with a slight abuse of notation, is defined from (1) when the players have critical thresholds $r_{A}$ and $r_{B}$. Let $T\left(r_{A}, r_{B}, \omega\right)$ be the time of the first adjustment given the thresholds $r_{A}$ and $r_{B}$, i.e., the first time after $t=0$ that the contract payment relative to adjustment payment is either equal or below $r_{A}$ or equal or above $r_{B}$, viz.,

$$
T\left(r_{A}, r_{B}, \omega\right)=\inf \left\{t>0 \mid R(t, \omega) / Z(t, \omega) \notin\left(r_{A}, r_{B}\right)\right\} .
$$

Given the thresholds, define the expected contribution to the objective function of player $\nu$ from the start at $t=0$ to the first contract adjustment,

$$
f_{\nu}\left(r_{A}, r_{B}\right)=E\left\{\int_{0}^{T\left(r_{A}, r_{B}\right)} R^{\eta_{\nu}}(s, \omega) \exp (-\beta s) d s\right\} .
$$

\footnotetext{
${ }^{3}$ Here and in the following we will write $E\{\Phi(a, b)\}$ for the expectation value of a stochastic variable $\Phi(a, b, \omega)$, rather then more cumbersome $E\{\Phi(a, b, \omega)\}$ or $E\{\Phi(a, b, \cdot)\}$.
} 


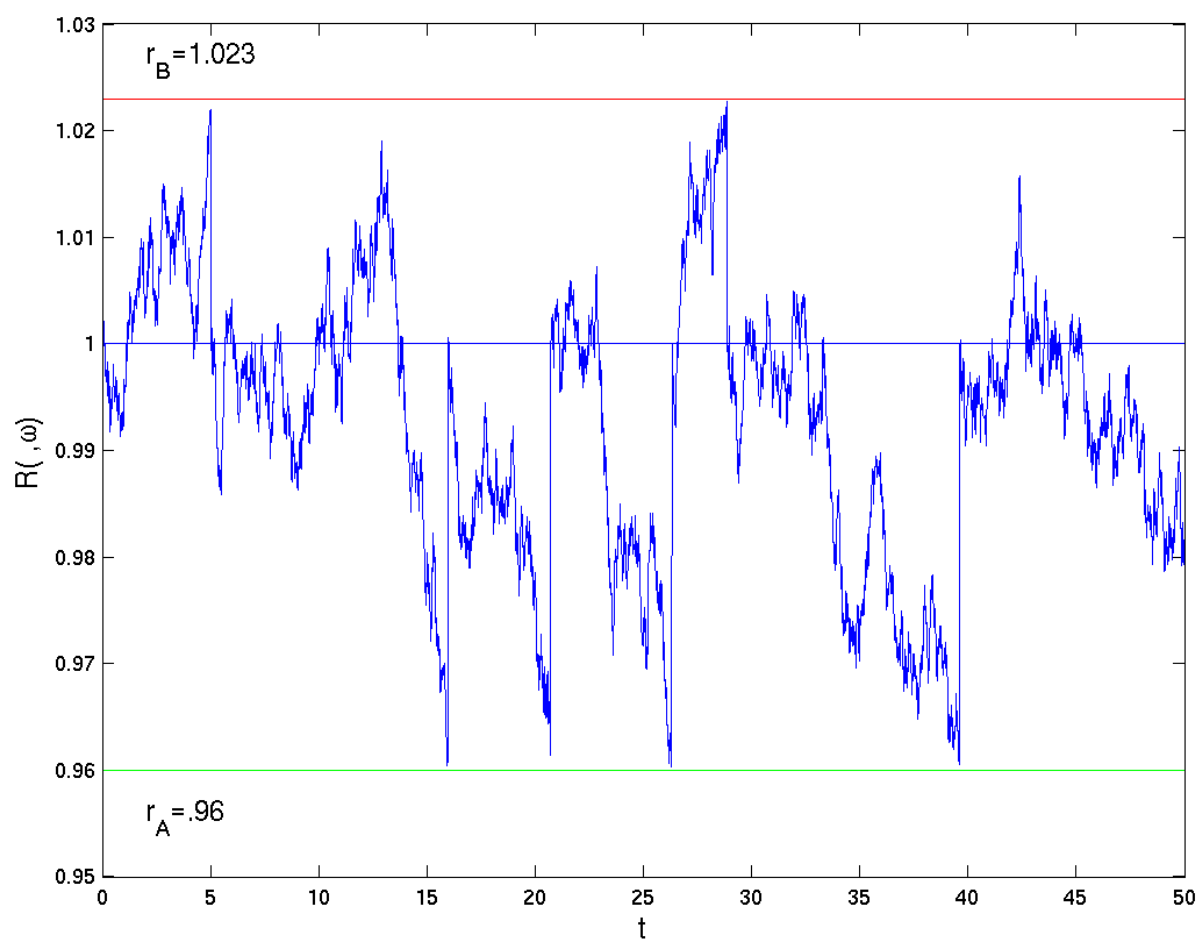

Figure 1. The figure shows one realization of the process $R(\cdot, \omega)$ in the case with $Z=1$ and $Q$ is geometric (or exponential) Brownian motion $Q=\exp \left(\left(\alpha_{q}-a_{q}^{2} / 2\right) t+a_{q} B_{t}\right)$ with drift $\alpha_{q}=.002$ and volatility $a_{q}=.01$. Here $B_{t}$ denotes standard Brownian motion. When the unit of time is interpreted as one month, this corresponds to $2.4 \%$ annual inflation. The process is sampled at 5000 points.

The expected flow payoff just after the first adjustment, discounted down to time $t=0$, is defined by

$$
h_{\nu}\left(r_{A}, r_{B}\right)=E\left\{Z^{\eta_{\nu}}\left(T\left(r_{A}, r_{B}\right)\right) \exp \left(-\beta T\left(r_{A}, r_{B}\right)\right)\right\} .
$$

Note that in the special case where the real adjustment outcome $Z$ is a constant, $h_{\nu}$ is a pure discount factor. Note also that the second inequality in Definition 2.2 ensures that $h_{\nu}<1$.

Then we may formulate the following theorem.

Theorem 3.1. Assume the real adjustment outcome $Z$ and the aggregate price $Q$ satisfy property $\mathcal{F}$. Furthermore, assume that both players use threshold strategies, implying that the contract is adjusted as soon as the contract payment relative to the adjustment outcome $R / Z$ exits the interval $\left(r_{A}, r_{B}\right)$. Then the following properties hold:

(i) The expected values of the objective functions immediately after an adjustment satisfy

$$
u_{\nu}\left(r_{A}, r_{B}\right)=\frac{f_{\nu}\left(r_{A}, r_{B}\right)-\tau_{\nu} h_{\nu}\left(r_{A}, r_{B}\right)}{1-h_{\nu}\left(r_{A}, r_{B}\right)}, \quad \nu=A, B
$$

and are defined for $0 \leq r_{A}<1<r_{B} \leq \infty$.

(ii) Assuming $f$ and $h$ are differentiable, the derivatives satisfy

$$
\frac{\partial u_{\nu}}{\partial r_{\mu}}=\frac{\frac{\partial f_{\nu}}{\partial r_{\mu}}+\left(u_{\nu}-\tau_{\nu}\right) \frac{\partial h_{\nu}}{\partial r_{\mu}}}{1-h_{\nu}}, \quad \nu=A, B, \quad \mu=A, B .
$$

We make the following observations: 
(I) To facilitate the interpretation of equation (5), one may compare with the deterministic case where the contribution $f_{\nu}$ is deterministic between adjustments, and where the real adjustment outcome $Z$ is a constant, implying that $h_{\nu}$ is a pure discount factor. Recalling the formula for the sum of an infinite geometric series $\left(a+k a+k^{2} a+\cdots=a /(1-k)\right.$ when $\left.|k|<1\right)$, we see that equation (5) is on the same form, where $a=f_{\nu}-\tau_{\nu} h_{\nu}$ is the payoff accumulated in the time interval between two adjustments, including the discounted adjustment costs, and $k=h_{\nu}$ is the discount factor.

(II) Equation (6) captures the opposing effects of increasing the thresholds: For example, increasing the threshold for player $\mathrm{A}, r_{A}$, reduces the expected time until the next adjustment. This will reduce the expected payoff until the next adjustment, i.e., $\frac{\partial f_{\nu}}{\partial r_{A}}<0$. Furthermore, reducing the expected time until the next adjustment raises the discount factor $\frac{\partial h_{\nu}}{\partial r_{A}}>0$, reflecting that the adjustment $\operatorname{cost} \tau_{\nu}$ is incurred earlier, but also that the value of the objective function after an adjustment $u_{\nu}$ is received earlier. The denominator reflects that in terms of expected values, all intervals between adjustments are identical, so the effect on payoff within one interval multiplies.

\section{NASH EQUILIBRIUM}

In order to prove the existence of a Nash equilibrium, we in this section impose the additional assumption that both the real adjustment outcome $Z$ and the aggregate price level $Q$ are continuous functions of time. This assumption implies that the Lévy measure vanishes, so that $Z$ and $Q$ reduce down to geometric Brownian motions. Define the expected value of the objective functions of each of the players, given optimal play by this player, as

$$
\begin{aligned}
& u_{m, A}\left(r_{B}\right)=\sup _{r_{A}} u_{A}\left(r_{A}, r_{B}\right), \\
& u_{m, B}\left(r_{A}\right)=\sup _{r_{B}} u_{B}\left(r_{A}, r_{B}\right) .
\end{aligned}
$$

Furthermore, define the optimal thresholds for player A, as a function of the threshold for player $\mathrm{B}, m_{A}\left(r_{B}\right)$, and similarly for player $\mathrm{B}, m_{B}\left(r_{A}\right)$, as follows

$$
\begin{aligned}
& m_{A}\left(r_{B}\right)=\inf \left\{r_{A} \in[0,1) \mid u_{A}\left(r_{A}, r_{B}\right)=u_{m, A}\left(r_{B}\right)\right\} \\
& m_{B}\left(r_{A}\right)=\sup \left\{r_{B} \in(1, \infty] \mid u_{B}\left(r_{A}, r_{B}\right)=u_{m, B}\left(r_{A}\right)\right\} .
\end{aligned}
$$

These definitions allow for the possibility that the optimal threshold is not unique, in which case they pick the most lenient value, i.e., the threshold farthest from unity. However, in Theorem 4.1 below, we show that when $Z$ and $Q$ are continuous, then the optimal threshold is indeed unique. If it is optimal for a player never to require contract adjustment, then we set $m_{A}\left(r_{B}\right)=0$ (player A) or $m_{B}\left(r_{A}\right)=\infty$ (player B).

When $Z$ and $Q$ are continuous, $f_{\nu}, h_{\nu}$, and hence $u_{\nu}$ are all continuous by Theorem 3.1. Then $u_{m, \nu}$ are continuous and $m_{\nu}$ are well-defined, piecewise continuous and $u_{A}\left(m_{A}\left(r_{B}\right), r_{B}\right)=$ $u_{m, A}\left(r_{B}\right)$ and $u_{B}\left(r_{A}, m_{B}\left(r_{A}\right)\right)=u_{m, B}\left(r_{A}\right)$. We may then state the following theorem regarding uniqueness of the optimal value and the existence of an equilibrium point.

Theorem 4.1. Assume the real adjustment outcome $Z$ and the aggregate price level $Q$ are geometric Brownian motions, satisfying property $\mathcal{F}$. Then the following properties hold:

(i) The expected value of the objective function for player $A, u_{A}\left(r_{A}, r_{B}\right)$, is increasing in the threshold of player $B, r_{B}$, i.e., $\frac{\partial u_{A}}{\partial r_{B}}>0$. The expected value of the objective function for player $B, u_{B}\left(r_{A}, r_{B}\right)$, is decreasing in the threshold of player $A, r_{A}$, i.e., $\frac{\partial u_{B}}{\partial r_{A}}>0$.

(ii) Given the threshold for player $B, r_{B}$, there exists a unique best response for player $A$, i.e., a unique value $0 \leq r_{A}=m_{A}\left(r_{B}\right)<1$ that maximizes $u_{A}\left(r_{A}, r_{B}\right)$. Correspondingly, given $r_{A}$, there exists a unique value $\infty \geq r_{B}=m_{B}\left(r_{A}\right)>1$ that maximizes $u_{B}\left(r_{A}, r_{B}\right)$.

(iii) If $m_{A}\left(r_{B}\right)>0$, then the function $m_{A}\left(r_{B}\right)$ is strictly increasing, and if $m_{B}\left(r_{A}\right)<\infty$, then the function $m_{B}\left(r_{A}\right)$ is strictly increasing.

(iv) If $m_{A}\left(r_{B}\right)>0$, then $m_{A}\left(r_{B}\right)$ is strictly decreasing in $\tau_{A}$. Correspondingly, if $m_{B}\left(r_{A}\right)<\infty$, 
then $m_{B}\left(r_{A}\right)$ is strictly increasing in $\tau_{B}$.

(v) There is at least one Nash equilibrium point $\left(r_{A}^{e}, r_{B}^{e}\right)$, where

$$
\begin{aligned}
& r_{A}^{e}=\operatorname{argmax}_{r<1}\left\{u_{A}\left(r, r_{B}^{e}\right)\right\}, \\
& r_{B}^{e}=\operatorname{argmax}_{r>1}\left\{u_{B}\left(r_{A}^{e}, r\right)\right\} .
\end{aligned}
$$

Theorem 4.1 ensures that both players have unique best response functions in the form of thresholds $r_{\nu}$. Furthermore, there exists a Nash equilibrium in thresholds. It is possible to prove existence of a Nash equilibrium under weaker assumptions than $Z$ and $Q$ being continuous. The essential criterion is that $m_{A}$ and $m_{B}$ are continuous. But this assumption leads to rather technical assumptions on $Z$ and $Q$.

Although we have not been able to construct cases with multiple Nash equilibria when $Z$ and $R$ are continuous, we have been unable to prove uniqueness of the Nash equilibrium in the general case. Thus, for each set of stochastic processes, it is necessary to verify that there is only one equilibrium point. Andersen and Christensen [2] prove that the equilibrium is unique in their model for a $\log$ Gaussian price.

As expected, Theorem 4.1 (iv), shows that higher adjustment costs make players more reluctant to require an adjustment, by pushing the threshold value for player $\mathrm{A}, m_{A}$, down, and for player $\mathrm{B}, m_{A}$, up, i.e., both further from unity. The interaction effects are, however, more interesting. First, part (i) shows that if one player becomes more aggressive (that is, has a threshold close to unity), this reduces the expected value of the objective function for the opponent. The opponent loses from both more frequent adjustment costs and on average a less favorable contract payment.

Second, and more important, Theorem 4.1(iii), identifies strategic substitutability in the choice of thresholds. This follows from the optimal thresholds $m_{A}\left(r_{B}\right)$ and $m_{B}\left(r_{A}\right)$ being increasing functions. If, in equilibrium, one player becomes more aggressive by choosing a threshold closer to unity, the other player becomes more passive by choosing a threshold further from unity. In other words, if, say, the adjustment fee of player B is reduced, making him more active, this will induce player A to become more passive. The intuition for this result is as follows. Demanding an adjustment involves an immediate cost, and then a gain by a more favorable contract payment until the next adjustment. If the opponent is aggressive, i.e., the threshold of the opponent is close to unity, the expected time until the next adjustment is short, so that the gain from a more favorable contract will be short-lived. In contrast, if the opponent is more passive, with a threshold further from unity, the gain from a more favorable contract is likely to last longer, making an adjustment more attractive.

The strategic substitutability effect is in contrast to Andersen and Christensen [3], who find strategic complementarity in the choice of thresholds. Their result appears to be due to the fact that they consider only one contract adjustment, implying an incentive for players to preempt the opponent. Thus, if one player is aggressive, the opponent has an incentive to also be aggressive, to increase the likelihood of being the player who obtains the advantage of asking for an adjustment at a suitable moment. Andersen and Christensen [2] consider the model with a finite, but large number of contract renewals, but it is not stated whether the strategic complementarity holds in that model.

Other observations include:

(I) If the nominal adjustment outcome $Z Q$ is monotone, then only one of the players will be active. The critical threshold of the other player is immaterial, thus, there is no unique optimal strategy for this player.

(II) The model may be generalized to the case where the adjustment $\operatorname{costs} \tau_{A}$ and $\tau_{B}$ depend on which player that requires contract adjustment. In equations (5) and (6), this would require that $\tau_{\nu}$ is replaced by the expected value of the contract adjustment fee, which again would be a function of $r_{A}$ and $r_{B}$. Theorem 4.1 is also valid in the generalized model, but in equations (12) and (13) in the proof and the calculations leading to these equations, $\tau_{A}$ must be interpreted as the contract adjustment when player A requires a contract adjustment. Similarly, in equation (14) $\tau_{B}$ must be interpreted as the contract adjustment when player B requires a contract adjustment. The model may also be generalized to allow for the adjustment fees being stochastic, where $\tau_{\nu}$ is the expected value of the adjustment fee. 
TABLE 1. The Nash equilibrium point for the example illustrated in Figures 2-4.

\begin{tabular}{cccccc}
$r_{A}^{e}$ & $r_{B}^{e}$ & $u_{A}\left(r_{A}^{e}, r_{B}^{e}\right)$ & $u_{B}\left(r_{A}^{e}, r_{B}^{e}\right)$ & $P\left(r_{A}^{e}, r_{B}^{e}\right)$ & $E\left\{T\left(r_{A}^{e}, r_{B}^{e}\right)\right\}$ \\
\hline .960 & 1.023 & 196.7 & 200.8 & .65 & 9.7 \\
\hline
\end{tabular}

(III) In special cases it is possible to find analytic expressions for some of the variables. Assume the real adjustment outcome relative to the real contract payment is given by a geometric Brownian motion $Z(t, \omega) / R(t, \omega)=Z(t, \omega) Q(t, \omega)=\exp \left(\left(\alpha-a^{2} / 2\right) t+a B_{t}(\omega)\right)$ where the notation is simplified by the normalization $Z(0, \omega) Q(0, \omega)=1$, and $B_{t}$ denotes the standard Brownian motion. Then (see Borodin and Salminen [4, p. 233, formula 3.0.1])

$$
E\left\{\exp \left(-\beta T\left(r_{A}, r_{B}\right)\right)\right\}=\left(r_{A}^{\gamma}\left(r_{B}^{\sigma}-r_{B}^{-\sigma}\right)-r_{B}^{\gamma}\left(r_{A}^{\sigma}-r_{A}^{-\sigma}\right)\right)\left(\left(r_{B} / r_{A}\right)^{\sigma}-\left(r_{B} / r_{A}\right)^{-\sigma}\right)^{-1}
$$

with $\gamma=\alpha a^{-2}-1 / 2$ and $\sigma=\sqrt{\gamma^{2}+2 \beta a^{-2}}$. By differentiating this expression with respect to $\beta$ at $\beta=0$ we find, where $\tilde{\sigma}=\sqrt{a^{2}+8 \beta} /(2 a)$,

$$
\begin{aligned}
& E\left\{T\left(r_{A}, r_{B}\right)\right\}= \frac{1}{a^{2} \gamma\left(r_{A} r_{B}\right)^{1 / 2}}\left(\left(\frac{r_{B}}{r_{A}}\right)^{\tilde{\sigma}}-\left(\frac{r_{B}}{r_{A}}\right)^{-\tilde{\sigma}}\right)^{-1} \\
& \times\left[\ln \left(r_{A}\right)\left(r_{A}^{\tilde{\sigma}+1 / 2}-r_{A}^{-\tilde{\sigma}+1 / 2}\right)-\ln \left(r_{B}\right)\left(r_{B}^{\tilde{\sigma}+1 / 2}-r_{B}^{-\tilde{\sigma}+1 / 2}\right)\right. \\
& \quad-\ln \left(r_{B} / r_{A}\right) \frac{\left(\frac{r_{B}}{r_{A}}\right)^{\tilde{\sigma}}+\left(\frac{r_{B}}{r_{A}}\right)^{-\tilde{\sigma}}}{\left(\frac{r_{B}}{r_{A}}\right)^{\tilde{\sigma}}-\left(\frac{r_{B}}{r_{A}}\right)^{-\tilde{\sigma}}} \\
&\left.\quad \times\left(\left(r_{A}^{\tilde{\sigma}+1 / 2}-r_{A}^{-\tilde{\sigma}+1 / 2}\right)+\left(r_{B}^{\tilde{\sigma}+1 / 2}-r_{B}^{-\tilde{\sigma}+1 / 2}\right)\right)\right]
\end{aligned}
$$

is the expected time to the first adjustment.

In Figures 2-4, we illustrate the model by presenting how key variables depend on the critical threshold $r_{A}$ and $r_{B}$, treating the thresholds as exogenous. Note that in almost all simulations, we include a positive drift in the aggregate price level $Q, \alpha_{q}>0$, representing inflation, implying a tendency that the real value of the contract payment, $R$, falls over time, relative to the real adjustment outcome, $Z$. Thus, it will usually be player A (the seller) who demands an adjustment, unless the critical threshold of player B is close to unity.

Figure 5 illustrates the game in setting thresholds. The curves show the best response functions $m_{A}$ and $m_{B}$ for different values of adjustment fees $\tau_{\nu}$, and the intersections indicate Nash equilibria. We observe that higher adjustment fee for one player leads to less aggressive play by this player, in the form of a threshold further from unity. The strategic substitutability effect is also apparent: reducing, say, the adjustment fee of player A, so that we consider thin curves further to the right, involves higher thresholds $r_{A}$ for player A, but in Nash equilibrium (represented by the intersections along one thick line), also higher thresholds for player B (i.e., lower values of $1 / r_{B}$, indicating more passive play). The strategic effect varies between the different cases, but in some cases it is rather strong.

For example, we see from Figure 5 that if we reduce the adjustment costs of player A, $\tau_{A}$, from .35 to .05 , keeping $\tau_{B}$ constant at $.35, r_{A}$ increases from .950 to .985 , implying that player A now requires an adjustment whenever he can increase the real contract payment by 1.5 percent, as opposed to a critical threshold of 5 percent before the change. Then the strategic effect implies that critical threshold of player B increases, from a threshold at 4 percent reduction in real contract payment to a threshold of 8.2 percent reduction $\left(1 / r_{B}\right.$ falls from .960 to .918$)$.

Comparison of Tables 2, 4-5 indicates that the threshold of player B is an increasing function of the drift in the aggregate price level. For example, for $\tau_{A}=\tau_{B}=0.05$, increasing the drift from $\alpha_{q}=0$ to $\alpha_{q}=0.006$ raises $r_{B}$ from 1.017 to 1.024 . This reflects that under high inflation (high drift), player B need not demand a adjustment even if he has been "unlucky" with the random movement, so that the contract payment is high relative to the adjustment payment. As the contract payment is set in nominal terms, high inflation will fairly soon reduce the real value of the contract payment, so player B need not incur the costs of an adjustment. In contrast, when the drift is weak (i.e., inflation low), player B must use a lower threshold to avoid lengthy periods 

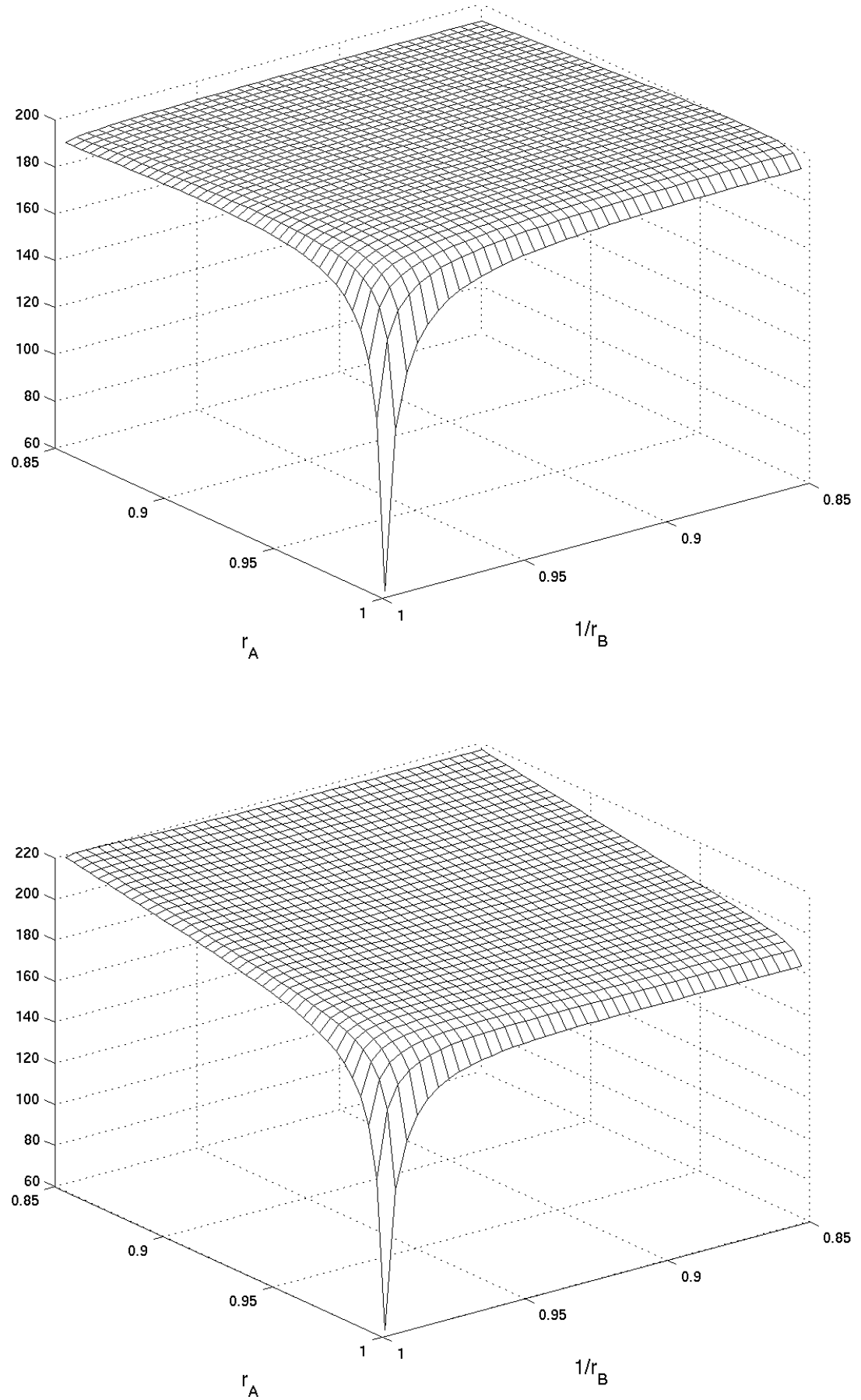

Figure 2. The expected discounted sum of flow payoffs of player A $u_{A}$ (top), and player $\mathrm{B}, u_{B}$ (bottom), for $r_{A}, 1 / r_{B} \in[.85,1]$ with the same process and parameters as in Figure 1. Furthermore, $\beta=.005, \eta_{A}=1, \eta_{B}=-1.5$, and $\tau_{A}=\tau_{B}=.1$. The plots are computed using $10^{5}$ realizations, each sampled at $2 \cdot 10^{5}$ points up to time 200 . Note that $u_{A}$ increases when $r_{B}$ increases. Because of the drift in the aggregate price level $Q$, there is a tendency that the real value of the contract payment falls over time, inducing player A to demand an adjustment. Thus, $r_{A}$ is more important for both $u_{A}$ and $u_{B}$ than $r_{B}$ is, except when $r_{B}$ is close to 1 . 


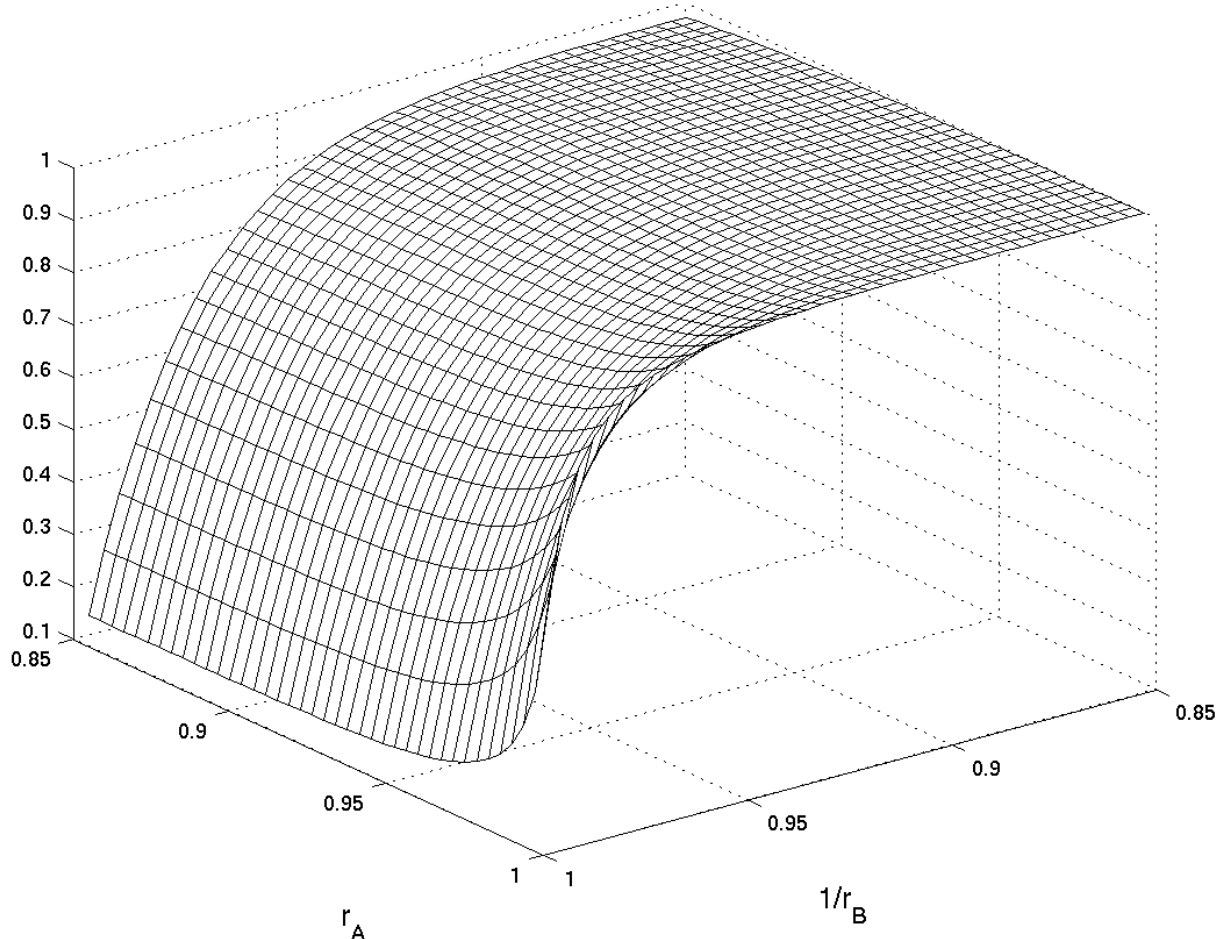

Figure 3 . The function $P\left(r_{A}, r_{B}\right)$ is the expected fraction of times the process reaches $r_{A}$ before it reaches $r_{B}$, as a function of $\left(r_{A}, r_{B}\right)$. Parameters and processes as in Figure 2 .

TABle 2. The Nash equilibrium points for various combinations of $\tau_{A}$ and $\tau_{B}$. Drift $\alpha_{q}=.002$ and volatility $a_{q}=.01$. Other parameters as in Figure 2.

\begin{tabular}{cccccccc}
$\tau_{A}$ & $\tau_{B}$ & $r_{A}^{e}$ & $r_{B}^{e}$ & $u_{A}\left(r_{A}^{e}, r_{B}^{e}\right)$ & $u_{B}\left(r_{A}^{e}, r_{B}^{e}\right)$ & $P\left(r_{A}^{e}, r_{B}^{e}\right)$ & $E\left\{T\left(r_{A}^{e}, r_{B}^{e}\right)\right\}$ \\
\hline .05 & .05 & .973 & 1.020 & 197.3 & 199.3 & .64 & 5.3 \\
.07 & .05 & .968 & 1.017 & 196.3 & 200.3 & .58 & 5.9 \\
.05 & .07 & .976 & 1.026 & 198.0 & 197.9 & .74 & 5.7 \\
.07 & .07 & .971 & 1.023 & 197.1 & 199.1 & .69 & 6.7 \\
.13 & .13 & .965 & 1.029 & 196.4 & 198.9 & .75 & 9.9 \\
.13 & .23 & .970 & 1.044 & 197.6 & 195.9 & .87 & 10.9 \\
.23 & .13 & .951 & 1.024 & 193.7 & 201.7 & .64 & 12.1 \\
.23 & .23 & .957 & 1.035 & 195.2 & 199.0 & .78 & 13.7 \\
\hline
\end{tabular}

TABLE 3. The Nash equilibrium points for various combinations of $\tau_{A}$ and $\tau_{B}$. Drift $\alpha_{q}=.002$ and volatility $a_{q}=.03$. Other parameters as in Figure 2.

\begin{tabular}{cccccccc}
$\tau_{A}$ & $\tau_{B}$ & $r_{A}^{e}$ & $r_{B}^{e}$ & $u_{A}\left(r_{A}^{e}, r_{B}^{e}\right)$ & $u_{B}\left(r_{A}^{e}, r_{B}^{e}\right)$ & $P\left(r_{A}^{e}, r_{B}^{e}\right)$ & $E\left\{T\left(r_{A}^{e}, r_{B}^{e}\right)\right\}$ \\
\hline .05 & .05 & .943 & 1.039 & 194.7 & 198.4 & .44 & 2.6 \\
.07 & .05 & .929 & 1.034 & 192.5 & 200.9 & .36 & 2.9 \\
.05 & .07 & .948 & 1.050 & 196.2 & 195.9 & .52 & 2.9 \\
.07 & .07 & .937 & 1.044 & 194.1 & 198.1 & .44 & 3.2 \\
.13 & .13 & .923 & 1.056 & 193.1 & 197.9 & .47 & 5.1 \\
.13 & .23 & .937 & 1.081 & 196.2 & 191.7 & .60 & 5.9 \\
.23 & .13 & .886 & 1.044 & 187.1 & 204.5 & .31 & 6.1 \\
.23 & .23 & .908 & 1.064 & 191.1 & 198.1 & .46 & 7.2 \\
\hline
\end{tabular}




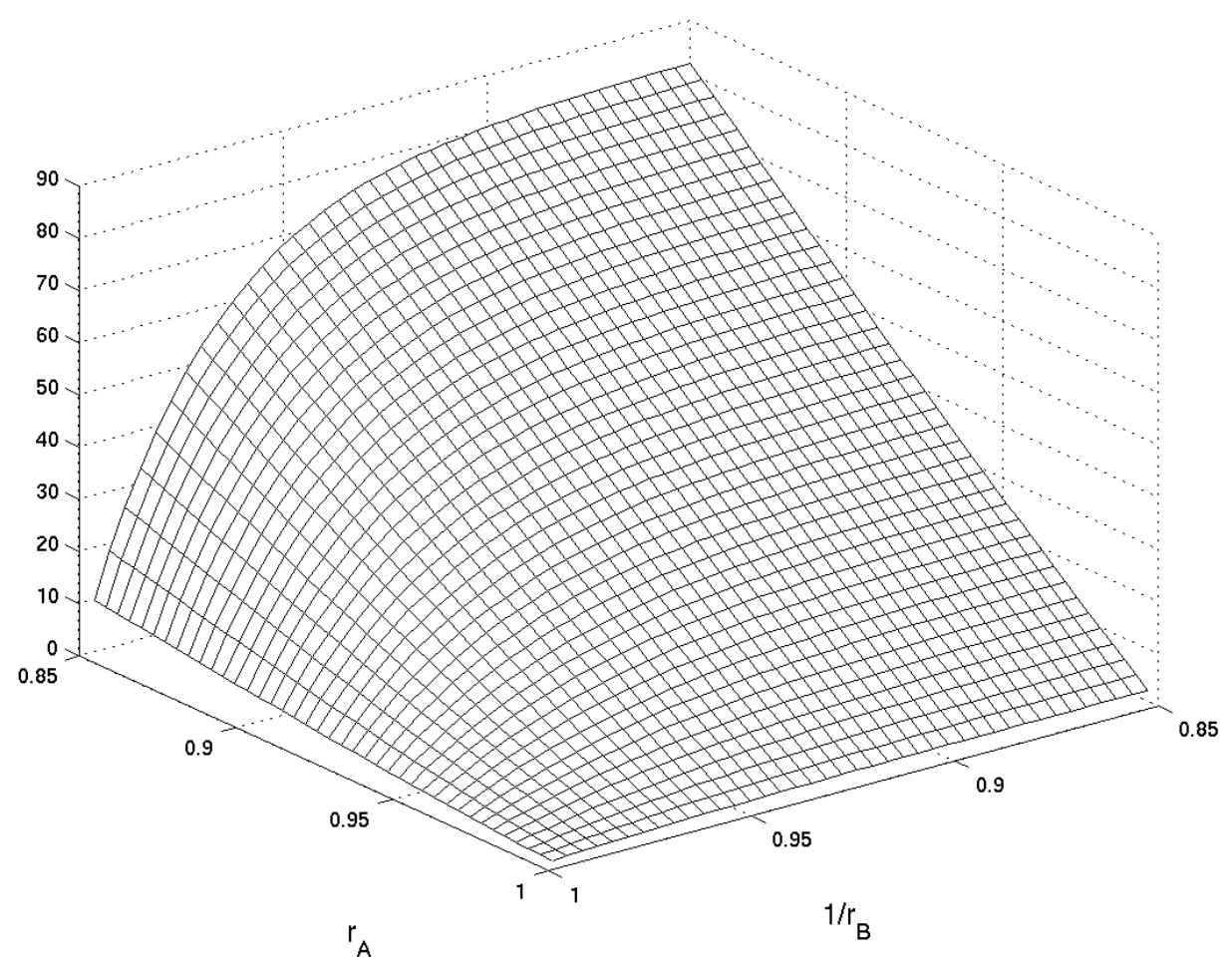

Figure 4. The function $E\left\{T\left(r_{A}, r_{B}\right)\right\}$ is the expected time until an adjustment, i.e., until the process leaves the interval $\left(r_{A}, r_{B}\right)$ the first time. Parameters and processes as in Figure 2.

TABLE 4. The Nash equilibrium points for various combinations of $\tau_{A}$ and $\tau_{B}$. Drift $\alpha_{q}=0$ and volatility $a_{q}=.01$. Other parameters as in Figure 2 .

\begin{tabular}{cccccccc}
$\tau_{A}$ & $\tau_{B}$ & $r_{A}^{e}$ & $r_{B}^{e}$ & $u_{A}\left(r_{A}^{e}, r_{B}^{e}\right)$ & $u_{B}\left(r_{A}^{e}, r_{B}^{e}\right)$ & $P\left(r_{A}^{e}, r_{B}^{e}\right)$ & $E\left\{T\left(r_{A}^{e}, r_{B}^{e}\right)\right\}$ \\
\hline .05 & .05 & .971 & 1.017 & 197.3 & 199.2 & .37 & 5.2 \\
.07 & .05 & .965 & 1.016 & 196.4 & 200.2 & .31 & 6.1 \\
.05 & .07 & .976 & 1.024 & 198.4 & 197.7 & .49 & 6.2 \\
.07 & .07 & .969 & 1.020 & 197.2 & 199.0 & .38 & 6.7 \\
.13 & .13 & .962 & 1.026 & 196.7 & 198.6 & .40 & 10.3 \\
.13 & .23 & .968 & 1.038 & 198.3 & 195.7 & .53 & 12.4 \\
.23 & .13 & .944 & 1.021 & 193.9 & 201.6 & .26 & 12.5 \\
.23 & .23 & .954 & 1.032 & 196.1 & 198.4 & .40 & 15.4 \\
\hline
\end{tabular}

TABLE 5. The Nash equilibrium points for various combinations of $\tau_{A}$ and $\tau_{B}$. Drift $\alpha_{q}=.006$ and volatility $a_{q}=.01$. Other parameters as in Figure 2 .

\begin{tabular}{cccccccc}
$\tau_{A}$ & $\tau_{B}$ & $r_{A}^{e}$ & $r_{B}^{e}$ & $u_{A}\left(r_{A}^{e}, r_{B}^{e}\right)$ & $u_{B}\left(r_{A}^{e}, r_{B}^{e}\right)$ & $P\left(r_{A}^{e}, r_{B}^{e}\right)$ & $E\left\{T\left(r_{A}^{e}, r_{B}^{e}\right)\right\}$ \\
\hline .05 & .05 & .973 & 1.024 & 196.3 & 199.4 & .95 & 4.0 \\
.07 & .05 & .968 & 1.020 & 195.2 & 200.7 & .92 & 4.7 \\
.05 & .07 & .974 & 1.032 & 196.5 & 198.2 & .97 & 4.1 \\
.07 & .07 & .969 & 1.028 & 195.6 & 199.5 & .97 & 4.9 \\
.13 & .13 & .960 & 1.034 & 193.6 & 200.0 & .98 & 6.7 \\
.13 & .23 & .960 & 1.054 & 193.8 & 196.8 & .99 & 6.7 \\
.23 & .13 & .945 & 1.028 & 190.9 & 203.3 & .96 & 8.9 \\
.23 & .23 & .947 & 1.043 & 191.3 & 200.6 & .99 & 8.9 \\
\hline
\end{tabular}




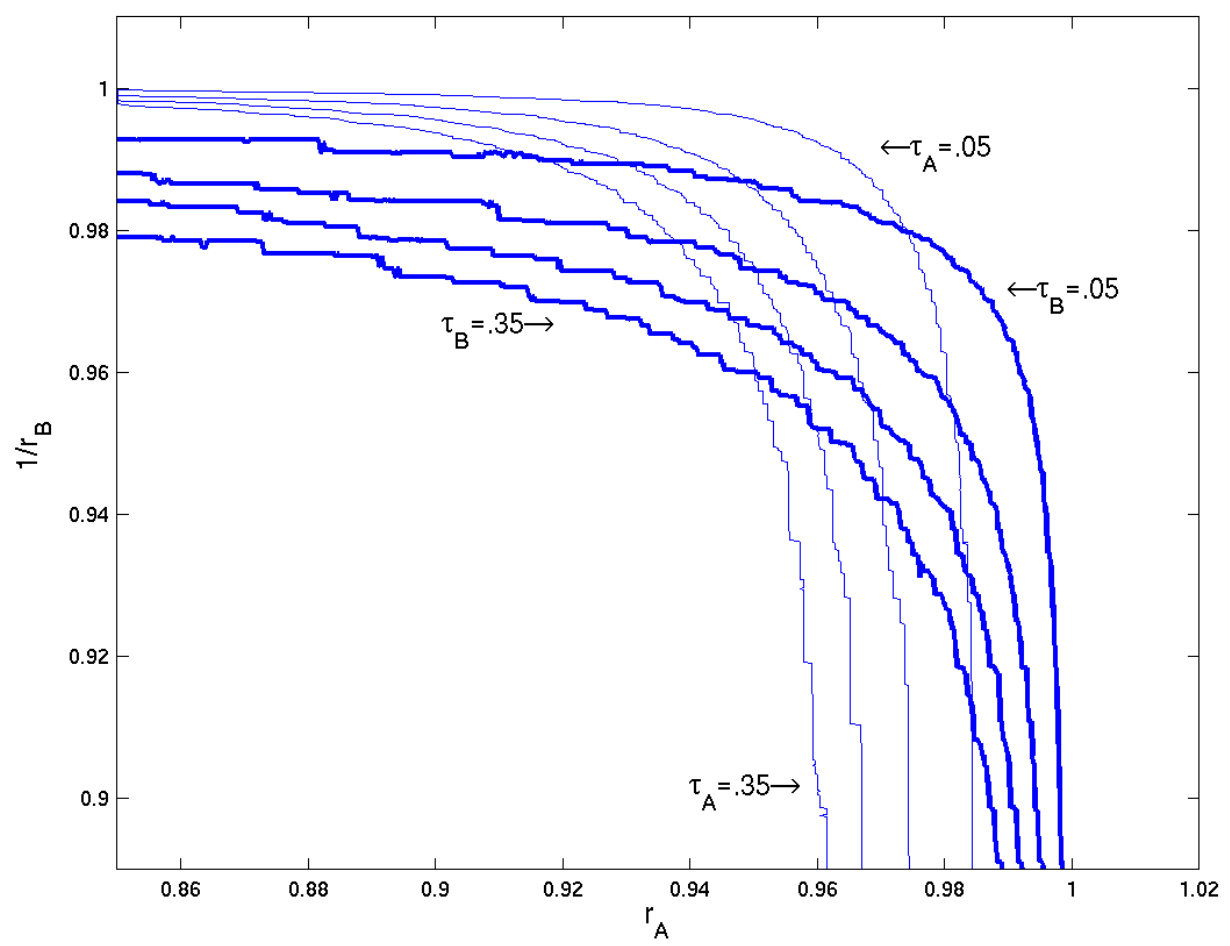

Figure 5. The best response function $m_{A}$ (thin curves) and $m_{B}$ (thick curves) of players $\mathrm{A}$ and $\mathrm{B}$, respectively, for values of $\tau_{A}$ and $\tau_{B}$ from .05 to .35 . Intersection between $m_{A}$ and $m_{B}$ gives the Nash equilibrium point $\left(r_{A}^{e}, r_{B}^{e}\right)$ for the particular set of $\left(\tau_{A}, \tau_{B}\right)$. Other parameters and processes are as in Figure 2.

TABLE 6. The Nash equilibrium points for various combinations of $\tau_{A}$ and $\tau_{B}$. Drift $\alpha_{q}=.002$, volatility $a_{q}=.01, \eta_{A}=.7$, and $\eta_{B}=-1.5$. Other parameters as in Figure 2 .

\begin{tabular}{cccccccc}
$\tau_{A}$ & $\tau_{B}$ & $r_{A}^{e}$ & $r_{B}^{e}$ & $u_{A}\left(r_{A}^{e}, r_{B}^{e}\right)$ & $u_{B}\left(r_{A}^{e}, r_{B}^{e}\right)$ & $P\left(r_{A}^{e}, r_{B}^{e}\right)$ & $E\left\{T\left(r_{A}^{e}, r_{B}^{e}\right)\right\}$ \\
\hline .05 & .05 & .968 & 1.018 & 197.4 & 200.3 & .59 & 6.0 \\
.07 & .05 & .960 & 1.016 & 196.5 & 201.7 & .52 & 7.0 \\
.05 & .07 & .971 & 1.023 & 197.9 & 199.1 & .68 & 6.6 \\
.07 & .07 & .964 & 1.020 & 197.0 & 200.5 & .61 & 7.4 \\
.13 & .13 & .955 & 1.024 & 196.3 & 200.8 & .67 & 11.2 \\
.13 & .23 & .962 & 1.039 & 197.4 & 197.7 & .82 & 12.7 \\
.23 & .13 & .937 & 1.019 & 194.0 & 204.3 & .55 & 14.1 \\
.23 & .23 & .947 & 1.030 & 195.4 & 201.2 & .72 & 16.1 \\
\hline
\end{tabular}

of a disadvantageous contract payment. This result is in contrast to the findings of Andersen and Christensen [3], where increased drift makes player B (the principal in their model) more aggressive. Their result is probably due to their assumption of only one renegotiation; if there is drift that is disadvantageous to player B, there will be less reason for player B to postpone an adjustment in the hope of a more favorable adjustment a later stage. Indeed, in Andersen and Christensen [2], it is shown that the effect of drift is ambiguous in the case where it is allowed for many renegotiations.

The threshold of player $\mathrm{A}$ is non-monotonic in the drift. For example, for $\tau_{A}=\tau_{B}=0.23$, increasing the drift from $\alpha_{q}=0$, via $\alpha_{q}=0.002$ to $\alpha_{q}=0.006$ yields $r_{A}$ equal to $0.954,0.952$ and 
TABLE 7. The Nash equilibrium points for various combinations of $\tau_{A}$ and $\tau_{B}$. Drift $\alpha_{q}=.002$, volatility $a_{q}=.01$, and $\eta_{A}=-\eta_{B}=1.0$. Other parameters as in Figure 2.

\begin{tabular}{cccccccc}
$\tau_{A}$ & $\tau_{B}$ & $r_{A}^{e}$ & $r_{B}^{e}$ & $u_{A}\left(r_{A}^{e}, r_{B}^{e}\right)$ & $u_{B}\left(r_{A}^{e}, r_{B}^{e}\right)$ & $P\left(r_{A}^{e}, r_{B}^{e}\right)$ & $E\left\{T\left(r_{A}^{e}, r_{B}^{e}\right)\right\}$ \\
\hline .05 & .05 & .976 & 1.027 & 198.0 & 198.5 & .75 & 5.8 \\
.07 & .05 & .971 & 1.023 & 197.0 & 199.3 & .68 & 6.6 \\
.05 & .07 & .979 & 1.036 & 198.9 & 197.3 & .85 & 6.4 \\
.07 & .07 & .974 & 1.030 & 197.7 & 198.3 & .77 & 7.2 \\
.13 & .13 & .967 & 1.038 & 197.1 & 198.0 & .83 & 10.7 \\
.13 & .23 & .973 & 1.061 & 198.5 & 195.2 & .94 & 11.6 \\
.23 & .13 & .956 & 1.032 & 194.9 & 199.8 & .75 & 13.5 \\
.23 & .23 & .963 & 1.051 & 196.6 & 197.4 & .90 & 15.2 \\
\hline
\end{tabular}

0.947, respectively. This reflects two opposing effects. On the one hand, stronger drift implies that for a given threshold, adjustments will be more frequent, so that the total costs incurred from adjustment increase. To reduce the rise in costs from frequent adjustments, player A will be more reluctant to demand an adjustment, thus the threshold is decreased. On the other hand, the strategic substitutability in the choice of thresholds implies that when higher drift increases the threshold of player B, making him less aggressive, it also increases the threshold of player A. Intuitively, the increasing threshold of player B raises the possible gain for player A of requiring an adjustment, in the hope of obtaining an advantageous evolution of the contract payment.

Comparing Tables 2-3 indicates that greater volatility makes both players more reluctant to require an adjustment, so that the threshold of player A decreases, and the threshold of player B increases, both further away from unity. The intuition is straightforward: with greater volatility, thresholds close to unity will imply too frequent adjustments, thus players are less aggressive so as to reduce adjustment costs. This result is the same as derived by Andersen and Christensen [3]. Note, however, that the change is not so large that it prevents that the expected time between adjustments, ET, falls.

Table 6 shows the effect of reducing $\eta_{A}$, making player A more risk averse. Comparing with Table 2, we see that the thresholds of both players are reduced, player A's further away from unity and player B's closer to unity. Thus, risk aversion makes player A more reluctant to require an adjustment, inducing player B to become more aggressive. We also see that player B obtains higher expected utility when player A is more risk averse, corresponding to the well-known result that it is advantageous to bargain with a risk averse player (see, e.g., Osborne and Rubinstein [21, p. 18]). Likewise, Table 7 shows the effect of reducing $\eta_{B}$, making player B less risk loving. This improves the situation for player A, as the threshold of both players increases, making player A more aggressive and player B more passive, resulting in an increase in the expected utility of player A. (Clearly, it is less relevant to consider the change in the expected utility for the player whose utility function changes.)

\section{Discontinuities in the Real adjustment payment $Z$ or the aggregate price $Q$}

In this section we consider the effects of discontinuities in $Z$ and $Q$. We show that in some specific cases, Nash equilibrium requires that one of the players uses a mixed strategy. The reason is that, if $Z$ or $Q$ are discontinuous and make occasional jumps, this may give discontinuities in the optimal responses $m_{\nu}$. In most cases, this will not affect the existence of a Nash equilibrium with thresholds because the discontinuities in the $m_{\nu}$ will usually be very small. However, under some circumstances jumps in $Z$ or $Q$ may imply that $m_{A}\left(r_{B}\right)$ and $m_{B}\left(r_{A}\right)$ do not intersect. Then there will be no Nash equilibrium with constant threshold strategies. However, there will exist a Nash equilibrium in mixed strategies, in the sense that one player randomizes between two threshold values. To illustrate this, we consider a stylized example where there is a possibility that the real adjustment outcome $Z$ may take a large fall, which may lead player B to require an adjustment to reduce the contract payment down to the new, low value of $Z$.

Example 5.1. Assume that the aggregate price level $Q$ is constant, except for sudden increases according to a high intensity Poisson process, where the size of the increases are "small" and 


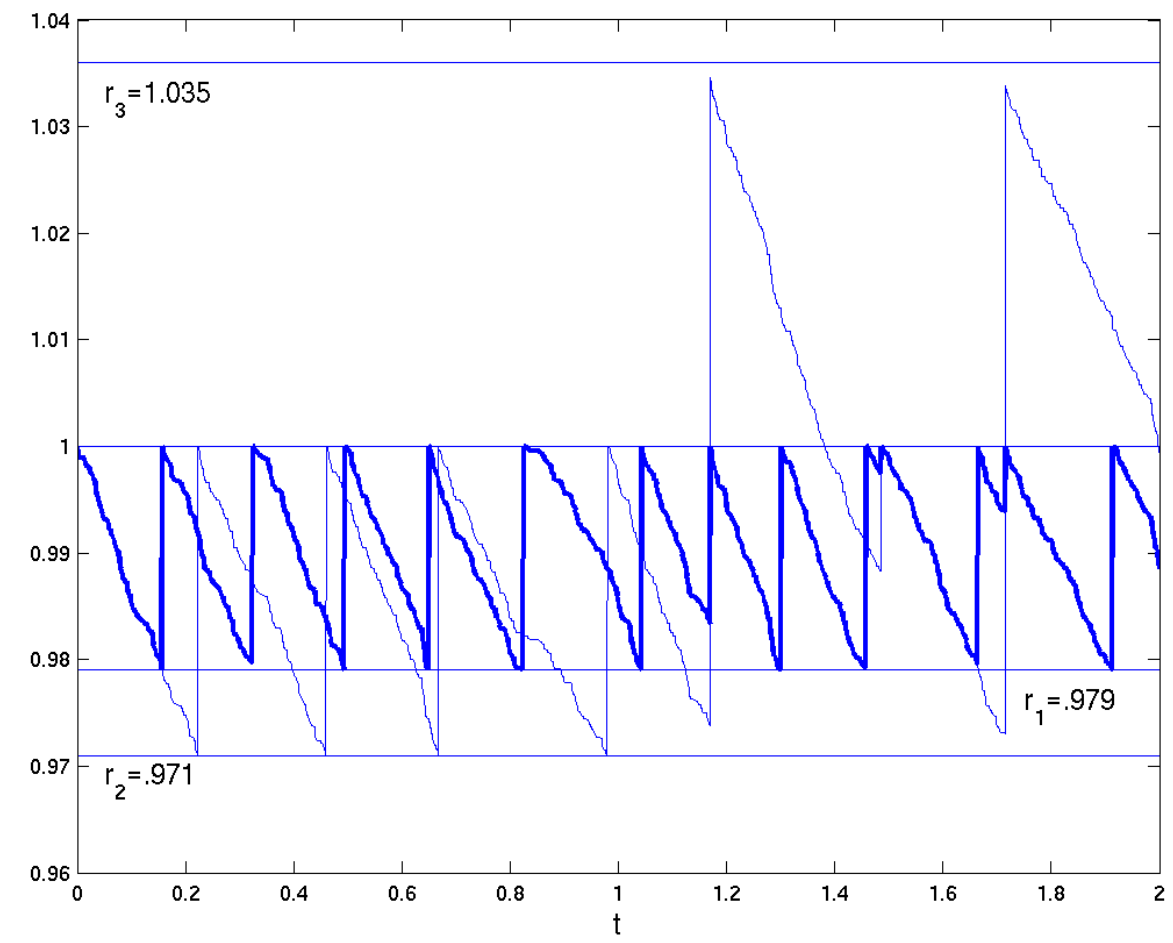

Figure 6 . Two realization of $R / Z$ for the same stochastic process, but corresponding to two different thresholds for player A, $r_{2}=.971$ and $r_{1}=.979$, in Example 5.1. Player $\mathrm{B}$ has threshold $r_{B}=r_{3}=1.035$. Threshold $r_{1}$ gives adjustment after every jump where $R / Z$ increases, while the lower threshold $r_{2}$ may give periods with high $R / Z$ values. In a Nash equilibrium, illustrated in Figure 7, player A randomizes between the thresholds $r_{2}$ and $r_{1}$. The two independent Lévy processes $Z$ and $Q$ are constants except for discrete changes according to a Poisson process. The Poisson process for $Z$ has intensity 8 and in the changes, $Z$ decreases with a factor 1.0625. The Poisson process for $Q$ has intensity approximately 1200 and in the changes, $Q$ increases according to a continuous distribution such that $E\{\ln (Q(t))\} \approx .13 t$ and $\operatorname{Var}\{\ln (Q(t, \omega))\} \approx .004 t$. Furthermore, $\tau_{A}=.0017$ and $\tau_{B}=.0057$ The process is modeled with time step .001 .

according to a continuous distribution. The real adjustment outcome $Z$ is also constant except for "large" discrete decreases at a fixed rate $1+\rho$, according to a low intensity Poisson process. These assumptions imply that the ratio of the contract payment relative to the adjustment outcome $R / Z$ is decreasing, except for sudden jumps where it increases with the percentage $\rho$.

Consider the situation if player B has chosen a threshold $r_{B}<1+\rho$. Then, if $Z$ jumps immediately after an adjustment, $R / Z$ will be above the critical threshold of player $\mathrm{B}$, inducing an immediate adjustment. Thus, player A will not benefit from a period with high $R / Z$ after the jump. On the other hand, if player A let $R / Z$ fall below $r_{B} /(1+\rho)$, a jump in $Z$ will nevertheless leave $R / Z$ below the threshold of the player $\mathrm{B}$. There will be no immediate adjustment, and player A will benefit from a period of high $R / Z$. This discontinuity at $r_{B} /(1+\rho)$ will imply a discontinuity in $m_{A}$, i.e., in the optimal threshold of player A.

When choosing the threshold, player $\mathrm{A}$ will have to weigh the loss of allowing a low $R / Z$ (by having a low threshold) against the possible gain of a period with high $R / Z$ if there is a fall in $Z$. However, if the threshold of player B is "rather low", maintaining the possibility of reaping a period of high $R / Z$ will require a very low threshold for player A. For sufficiently low threshold of player B, player A will then profit from neglecting the opportunity to benefit from a fall in $Z$. At 
that point, the optimal threshold of player A will be discontinuous; it will make a jump, as there is now no gain to be reaped by having a low threshold.

The situation is illustrated in Figures 6 and 7. In equilibrium, player A randomizes between two thresholds $r_{1} \approx .979$ and $r_{2} \approx .971$. Figure 6 shows that $R / Z$ falls monotonically, except when it increases to unity at an adjustment, or increases above unity when $Z$ falls. Figure 7 illustrates the strategic effects. For values of $r_{B}$ above $r_{3} \approx 1.035 \approx 1 / .966$, the optimal threshold of player A, $r_{A}$ is increasing in $r_{B}$ due to the strategic effect discussed in Section 4 . For $r_{B}<r_{3} \approx 1.035$, a fall in $Z$ will always induce player B to adjust, implying that player A sets $r_{A} \approx .979$. However, for $r_{B}=r_{3} \approx 1.035$, player $\mathrm{A}$ is indifferent between choosing a low threshold $r_{1}$, maintaining the possibility of benefiting from a fall in $Z$, and a high threshold $r_{2}$, which removes this possibility. In equilibrium, player $A$ mixes between these two thresholds, with probabilities ensuring that it is indeed optimal for player $\mathrm{B}$ to choose the threshold $r_{B}=r_{3} \approx 1.035$.

Let us now consider the consequences of discontinuities in $Z$ and $Q$ more formally. Define $S_{\nu}$ as the class of strategies for a player $\nu$, where the player randomizes between two thresholds $r_{1}$ and $r_{2}$, where $r_{1}$ is chosen with probability $1-q$ and $r_{2}$ with probability $q$. Note that $S_{\nu}$ includes pure strategies, where $q=0$. Let $s_{\nu} \in S_{\nu}$ denote a strategy. Furthermore, we assume that each time $R / Z$ is equal to unity or jumps from one side of unity to the other side of unity (either because the aggregate price or the real adjustment outcome fluctuates, or because an adjustment has taken place), players select one of the two thresholds at random. This procedure ensures that past fluctuations of $Z$ and $Q$ have no impact on the probability each player perceives of the thresholds of the opponent.

We extend the definition of the expected values of the objective functions $u_{\nu}$ to allow for randomization by both players. Furthermore, we define the optimal threshold for each player when the opponent randomizes:

$$
\begin{aligned}
& m_{A}^{c}\left(s_{B}\right)=\inf \left\{r_{A} \in[0,1) \mid u_{A}\left(r_{A}, s_{B}\right)=\sup _{r} u_{A}\left(r, s_{B}\right)\right\}, \\
& m_{B}^{c}\left(s_{A}\right)=\sup \left\{r_{B} \in(1, \infty] \mid u_{B}\left(s_{A}, r_{B}\right)=\sup _{r} u_{B}\left(s_{A}, r\right)\right\} .
\end{aligned}
$$

Thus, the function $m_{B}^{c}\left(s_{A}\right)$ corresponds to the usual optimal response function for player $\mathrm{B}$, $m_{B}\left(r_{A}\right)$, if player A uses a pure strategy. However, $m_{B}^{c}\left(s_{A}\right)$ is also defined if player A randomizes between thresholds $r_{1}$ and $r_{2}$, reflecting a discontinuity in $m_{A}\left(r_{B}\right)$. If $m_{B}^{c}\left(s_{A}\right)$ changes continuously from $m_{B}\left(r_{1}\right)$ to $m_{B}\left(r_{2}\right)$ when $q$ changes from 0 to 1 , we say that $m_{B}^{c}\left(s_{A}\right)$ is continuous. Continuity of the function $m_{A}^{c}\left(s_{B}\right)$ is defined similarly.

A Nash-equilibrium point is a pair of strategies $\left(s_{A}^{e}, s_{B}^{e}\right)$ with $s_{A}^{e} \in S_{A}$ and $s_{B}^{e} \in S_{B}$ where

$$
\begin{aligned}
& s_{A}^{e}=\operatorname{argmax}_{s_{A} \in S_{A}}\left\{u_{A}\left(s_{A}, s_{B}^{e}\right)\right\}, \\
& s_{B}^{e}=\operatorname{argmax}_{s_{B} \in S_{B}}\left\{u_{B}\left(s_{A}^{e}, s_{B}\right)\right\} .
\end{aligned}
$$

In order to prove existence of a Nash equilibrium, we assume that $m_{\nu}$ is piecewise continuous and that $m_{\nu}^{c}$ is continuous in each of the discontinuities in $m_{\nu}$. This is a property of the stochastic processes $Z$ and $Q$, but it will be fulfilled except in extreme cases. For example, it will not be fulfilled if $Z$ or $Q$ only take discrete values. ${ }^{4}$ We may then formulate the more general theorem for the existence of a Nash equilibrium.

Theorem 5.2. Assume the real adjustment outcome $Z$ and the aggregate price $Q$ satisfy property $\mathcal{F}$, that $m_{\nu}$ is piecewise continuous and that $m_{\nu}^{c}$ is continuous in each of the discontinuities in $m_{\nu}$. Then there exists at least one Nash-equilibrium point in which at most one player randomizes.

When the stochastic processes are discontinuous, we are able to construct examples where there exist multiple Nash equilibria. In Figure 8, there are two Nash equilibria with constant thresholds, and one with randomization.

\footnotetext{
${ }^{4}$ If $\operatorname{Prob}(Z(t, \omega)>r)$ and $\operatorname{Prob}(Q(t, \omega)>r)$ are continuous in $r$ for all values of $t$, then the functions $f_{\nu}, h_{\nu}$ are also continuous. Furthermore, $u_{\nu}$ is continuous by Theorem 3.1, $u_{m, \nu}$ is continuous and $m_{\nu}$ is well-defined, piecewise continuous and $u_{A}\left(m_{A}\left(r_{B}\right), r_{B}\right)=u_{m, A}\left(r_{B}\right)$ and $u_{B}\left(r_{A}, m_{B}\left(r_{A}\right)\right)=u_{m, B}\left(r_{A}\right)$.
} 


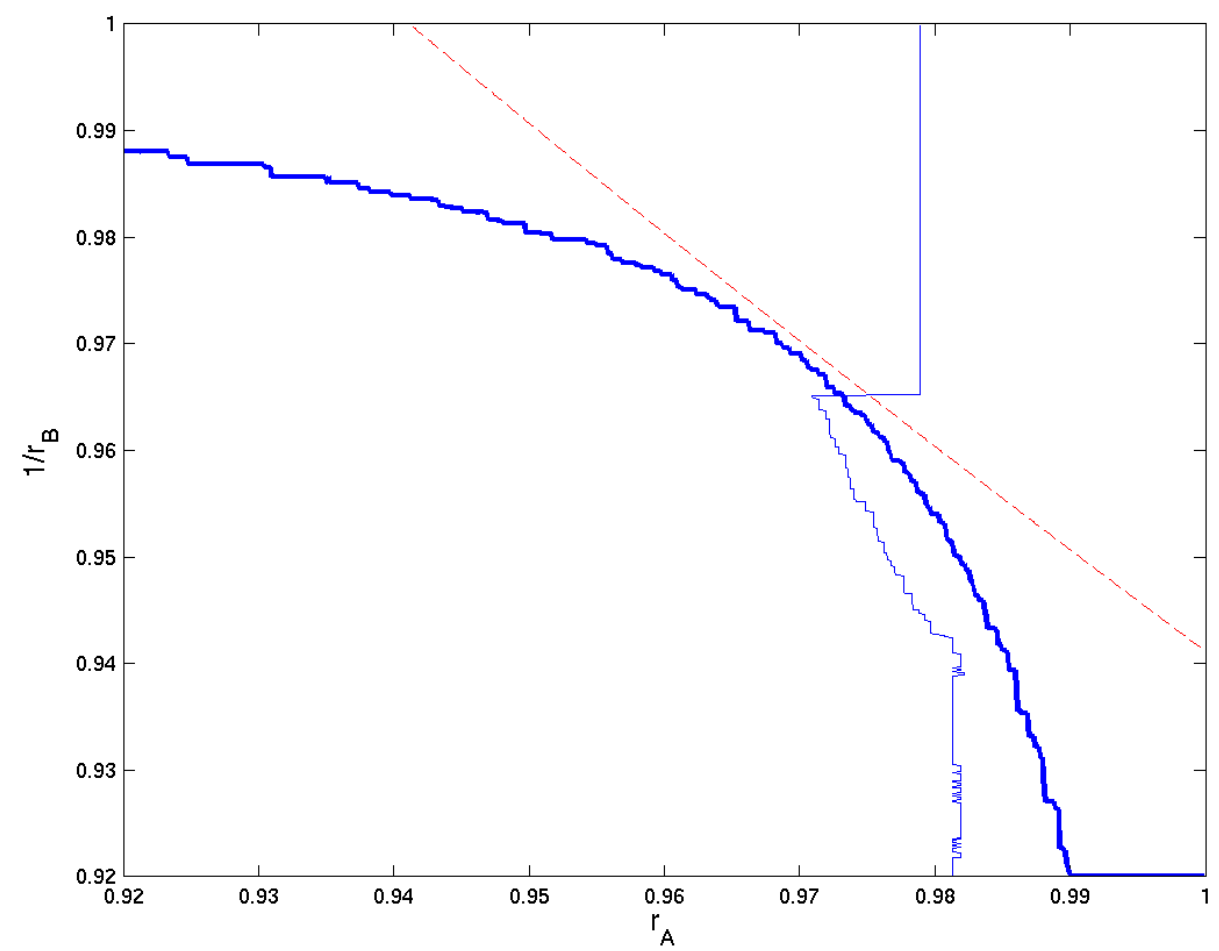

Figure 7 . The best response functions $m_{A}\left(R_{B}\right)$ (thin curve) and $m_{B}\left(R_{A}\right)$ (thick curve)corresponding to Example 5.1, for $r_{A}, 1 / r_{B} \in[.92,1]$. The dashed curve is $r_{B}=1.0625 r_{A}$. When the thresholds satisfy $r_{B}<1.0625 r_{A}$, there is an adjustment after every jump in $Z$. Thus, within this interval $r_{B}$ is immaterial, implying that $m_{A}\left(r_{B}\right)$ is vertical above the dashed curve. In equilibrium, player A's strategy $s_{A}$ implies randomization between the thresholds $r_{2} \approx .971$ and $r_{1} \approx .979$, the endpoints of the horizontal line in $m_{A}$ for $r_{B} \approx 1.035 \approx 1 / .966$. The curve $\left(E\left\{r_{A}\left(s_{A}\right)\right\}, m_{B}^{c}\left(s_{A}\right)\right)$ intersects the horizontal line in $m_{A}$ at $r_{A}=r^{\prime} \approx .973$. In a Nash equilibrium, player A selects the threshold $r_{1}$ with probability $\left(r^{\prime}-r_{2}\right) /\left(r_{1}-r_{2}\right) \approx .25$ and else $r_{2}$. The randomization makes the optimal threshold for player $\mathrm{B}$ equal to $r_{B} \approx 1.035 \approx 1 / .966$. $R$ and $Z$ are as defined in Figure 6 and other constants are $\beta=.005, \eta_{A}=1$, and $\eta_{B}=-1.5$. The plot is based on $10^{5}$ realizations, each sampled at $10^{5}$ points up to time 100 .

\section{EFFiciency of the CHOICE OF ADJUSTMENT COSTS}

In this section, we extend the model by allowing an additional stage of the model, taking place ahead of the basic model, where each of the players may invest in adjustment capacity, reducing his costs of adjustment of the contract. For example, a firm may have a large salary department, taking care of the wage negotiations. Let the costs of obtaining adjustment fee $\tau_{\nu}$ be given by the function $c_{\nu}\left(\tau_{\nu}\right)$, where we assume that $c_{\nu}$ is differentiable and strictly decreasing, and that $c_{\nu}$ converges to infinity when $\tau_{\nu}$ converges to zero, and $c_{\nu}$ converges to zero when $\tau_{\nu}$ converges to infinity. For simplicity, we assume that $c_{\nu}$ approaches zero sufficiently fast when $\tau_{\nu}$ increases to ensure that equations (7) and (8) below have a solution.

With a slight abuse of notation, let $W_{\nu}\left(\tau_{A}, \tau_{B}\right)$ denote the expected value of the objective function of player $\nu$, derived from Nash equilibrium in the basic model with adjustment fees $\tau_{A}$ and $\tau_{B}$. (We do not wish to go into issues of equilibrium selection here, so if there are multiple Nash equilibria, we assume that players observe a signal indicating which equilibrium applies. 


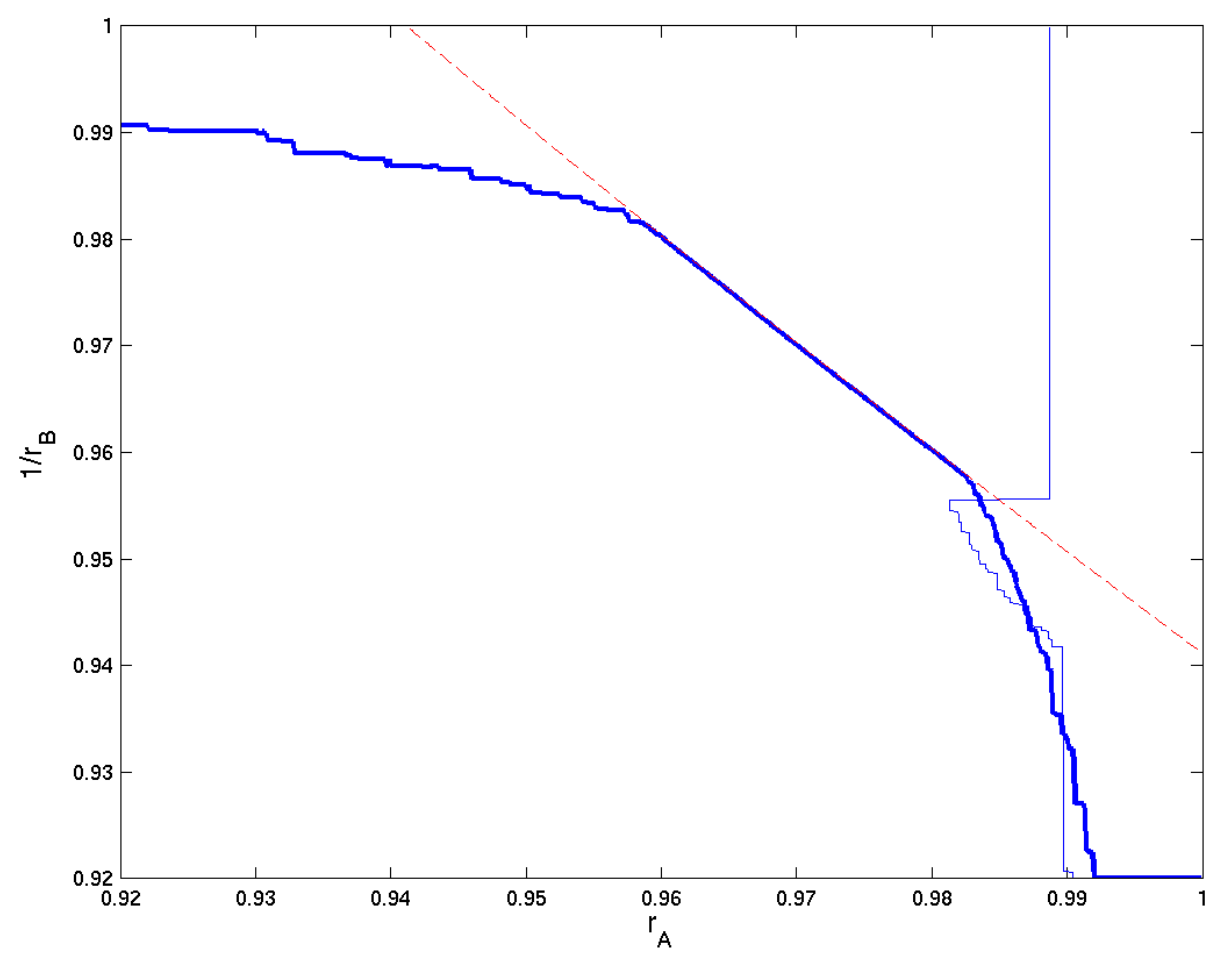

Figure 8. This figure shows non-uniqueness in the Nash equilibrium due to multiple crossings of the best response curves $m_{A}$ (thin curve) and $m_{B}$ (thick curve) for the process illustrated in Figure 7 with $\tau_{A}=.0005$ and $\tau_{B}=.0045$.

Associating probabilities with the various Nash equilibria, players take the expected value of the objective functions.)

When both players optimize their investment in adjustment capacity, the adjustment fees are given by the first order conditions

$$
\frac{\partial W_{\nu}}{\partial \tau_{\nu}}-c_{\nu}^{\prime}\left(\tau_{\nu}\right)=0, \quad \nu=A, B
$$

Assuming for simplicity that overall welfare can be measured by the sum of players' expected utility, the welfare maximizing levels of investment in adjustment capacity is given by

$$
\frac{\partial W_{A}}{\partial \tau_{\nu}}+\frac{\partial W_{B}}{\partial \tau_{\nu}}-c_{\nu}^{\prime}\left(\tau_{\nu}\right)=0, \quad \nu=A, B .
$$

From the fact that $\partial W_{A} / \partial \tau_{B}>0$ and $\partial W_{B} / \partial \tau_{A}>0$, it follows that the values of $\tau_{\nu}$ that satisfy (7) give positive values when put into the left-hand side of the equations (8). This implies that for each solution of (7), there exists a solution of (8) with higher values of $\tau_{\nu}$. This implies that when each player determines the adjustment fee from (7), there is an over-investment in adjustment capacity compared to a solution of equations (8).

This over-investment in adjustment capacity is due to the following. First, each of the players do not take into consideration that the contract payment in our setting is only a matter of a transfer between the players, so that what one player gains by adjusting the payment is directly linked to what the other player loses. Second, the first effect is exacerbated by the strategic substitutability in the choice of thresholds. By investing in adjustment capacity, thus reducing the adjustment fee, the threshold of the player is moved closer to unity, leading the other player to choose a threshold 


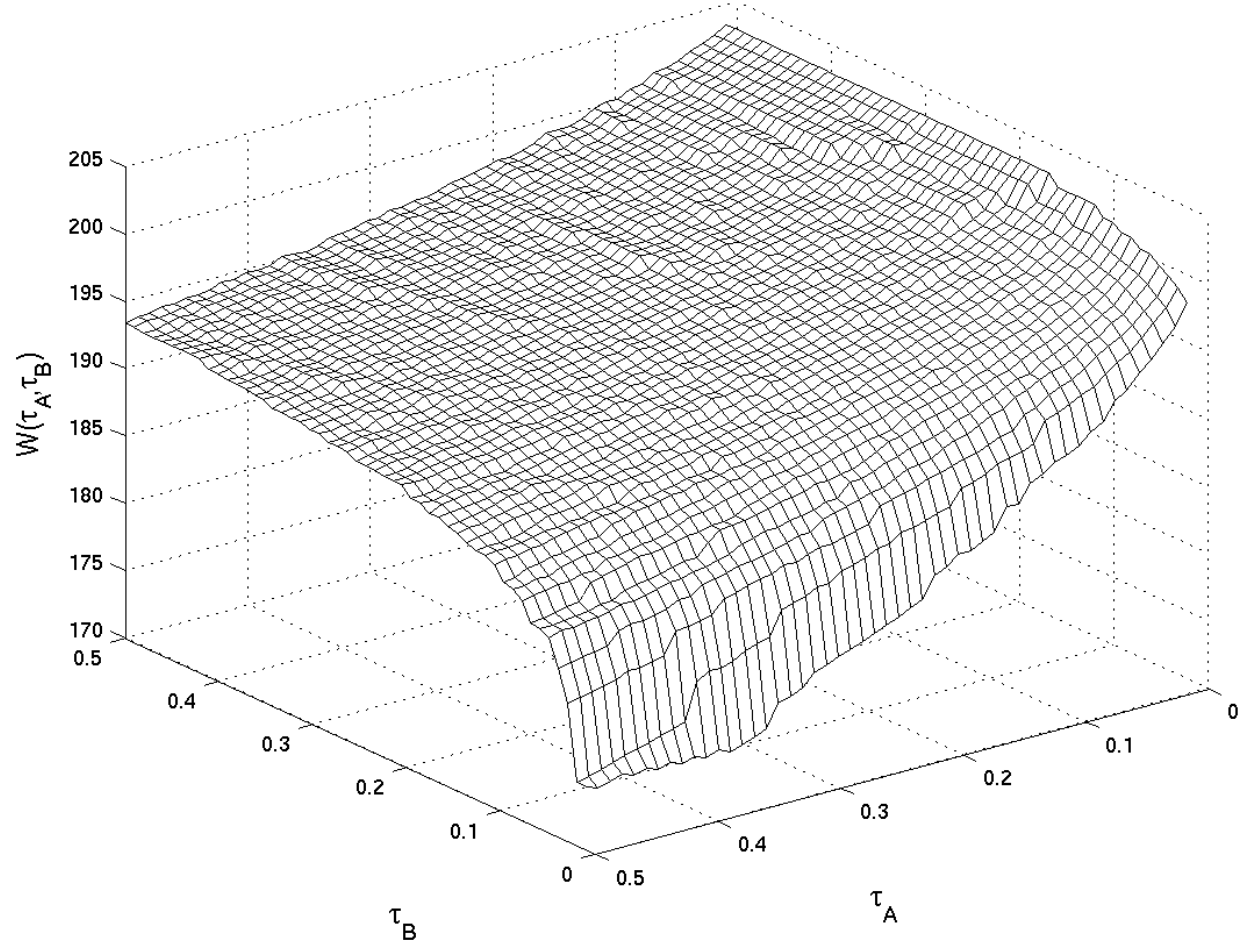

Figure 9. The figure shows the function $W_{A}\left(\tau_{A}, \tau_{B}\right)$ for $\tau_{A}, \tau_{B} \in[.01, .5]$. The data used are the same as in Table 2 and Figure 2.

further away from unity. The player gains from both changes, i.e., both from lowering his own adjustment fee, and from inducing the opponent to set a threshold further away from unity.

\section{COnCluding Remarks}

The assumption that wages and prices are sticky in nominal terms plays a key role in macro and monetary economics. However, usually the timing of price adjustment is taken as exogenous. This has motivated a considerable literature studying the optimal adjustment of prices under stochastic evolution of money or aggregate prices. In this paper we extend this analysis by considering bilateral adjustment, where both parties to the trade, both the seller and buyer, are allowed to require adjustments of the contract. This follows Andersen and Christensen [3], but they focus on only one adjustment, while we consider much more general stochastic processes, with an infinite horizon allowing for an unlimited number of adjustments.

We show that several of the key results from the literature on unilateral price adjustment also hold in the more general case of bilateral adjustment. Optimal behavior is characterized by threshold strategies, where players demand adjustment whenever they can improve the contract terms by a certain percentage, i.e., whenever the real contract payment, $R$, is too far away relative to the outcome of an adjustment of the contract, $Z$. Player A adjusts whenever $R / Z \leq r_{A}<1$, while player $\mathrm{B}$ requires adjustment whenever $R / Z \geq r_{B}>1$. As expected, higher volatility and larger adjustment costs make players more reluctant to demand an adjustment, implying threshold values further from unity.

Furthermore, we prove under rather general assumptions the existence of a Nash equilibrium in thresholds. A main result is that in equilibrium, there is strategic substitutability in players' choice of threshold: If one player becomes more aggressive, setting a threshold closer to unity, the other player becomes more passive, setting a threshold further from unity. The strategic substitutability 
may exacerbate asymmetries. For example, if the adjustment costs of player A are reduced, player A will respond by raising the threshold closer to unity. However, this effect will be strengthened by player B raising his threshold, further away from unity. Numerical simulations indicate that the strategic effect may be substantial. We also find that a risk averse player will be more passive, setting a threshold further from unity, thus benefiting the opponent.

One possible implication of the strategic substitutability is that it suggests that wages and prices set in bilateral bargaining are more sticky than wages and prices that are set unilaterally by one party. Unilateral price or wage setting may be seen as a special case of our model where the opponent is entirely passive. Thus, the strategic substitutability effect suggests that a price or wage setting firm will be more aggressive, in the sense of having a threshold closer to unity, than a firm which is linked to an opponent who may also adjust the price or wage. Typically, one would expect bilateral bargaining to be more frequent for wages than for prices, suggesting that wages stickiness might be more prevalent than price stickiness. ${ }^{5}$

We extend the basic model by introducing a stage prior to the model, where players may invest in "adjustment ability", in the sense that they may reduce their own costs of undertaking an adjustment (e.g., by having a personnel department doing the wage negotiations). We then find that players will over-invest as compared to the socially efficient level. The over-investment arises for two reasons. First, players require an adjustment too often from a social point of view, as they do not take into consideration that their own gain from better contract terms is reflected in a loss by the opponent. By investing to lower one's own adjustment costs, a player will require an adjustment more often, thus hurting the other player. Secondly, the strategic substitutability mentioned above exacerbates the first effect. By reducing one's own adjustment costs, a player becomes more aggressive. This makes the opponent more passive, which adds to the gain of the first player, as an adjustment requested by the opponent becomes less likely.

Our analysis is cast in a specific, but important setting, namely state-dependent adjustment of a nominal contract between two players, e.g., a labor contract or a tenancy agreement. However, we believe that our analysis also has wider applications. For example, the gains from trade between two countries may depend on the trade agreement that prevails between the countries. If one country perceives adjustment of the agreement as less costly than the other, the former country will be more aggressive in the sense of being willing to adjust the agreement even when only a small improvement is possible. Our finding of strategic substitutability in thresholds suggests that such asymmetry will be exacerbated, in the sense that the aggressive behavior of one country will make the other country more passive. This exacerbating of asymmetries resembles the finding of Haller and Holden [12], where it is shown that asymmetries in the bargaining positions of two countries over an international treaty may be exacerbated by the country with the "stronger" bargaining position setting a stricter super-majority ratification requirement, thus magnifying the effect of the strong bargaining position.

\section{Appendix A. Approximate Formulas}

Given the weak assumptions we impose on the stochastic processes, explicit formulas are difficult to obtain. However, we can derive some approximate formulas that may provide useful intuition for how the model works, and to get some sense of the numerical magnitudes that are involved.

We will first explore the effect on the payoff of a player from a marginal reduction in his threshold. Let players A and B have threshold $r_{1}>1$ and $r_{B}<\infty$. Consider the situation at $T_{1}$ when $R\left(T_{1}, \omega\right) / Z\left(T_{1}, \omega\right)=r_{1}$. If player A sticks to the threshold $r_{1}$, there will be an immediate adjustment at $T_{1}$. In contrast, if player $\mathrm{A}$ adopts a new threshold $r_{2}<r_{1}$, there will be an adjustment at $T_{2}$, where $T_{2}$ denotes the first time after $T_{1}$ where $R / Z$ has decreased at least by a factor $r_{2} / r_{1}$, or increased at least by a factor $r_{B} / r_{1}$. Formally

$$
T_{2}=\inf \left\{s>0 \mid R\left(T_{1}+s, \omega\right) / Z\left(T_{1}+s, \omega\right) \notin\left(r_{2}, r_{B}\right)\right\} .
$$

\footnotetext{
${ }^{5}$ However, the degree of stickiness varies among different prices, and there are also other differences between wage and price setting. Empirically, the relative stickiness of nominal wages versus nominal prices is disputed; see discussion in Spencer [24].
} 
Considering the payoffs associated with selecting the threshold $r_{2}$, when we let $r_{2}$ converge towards $r_{1}$ from below, we obtain the effect of a marginal reduction in $r_{1}$. The limit of the ratio of $R / Z$ is then

$$
v_{A}\left(r_{1}, r_{B}\right)=\lim _{r_{2} \rightarrow r_{1}-} \frac{E\left\{\int_{0}^{T_{2}} R^{\eta_{A}}\left(T_{1}+s\right) \exp (-\beta s) d s\right\}}{E\left\{\int_{0}^{T_{2}} Z^{\eta_{A}}\left(T_{1}+s\right) \exp (-\beta s) d s\right\}} .
$$

Define $v_{B}\left(r_{A}, r_{1}\right)$ correspondingly.

Assume there is a Gaussian component in either $Z$ or $Q$, i.e., that either $a_{z}>0$ or $a_{q}>0$. Then a well-known property of Gaussian processes implies that when $r_{1}$ is reached, the probability of reaching $r_{B}$ before $r_{2}$, converges to zero when $r_{2} \rightarrow r_{1}-$. Furthermore, the expected time until $r_{2}$ is reached converges to zero, i.e.,

$$
\lim _{r_{2} \rightarrow r_{1}-} E\left\{T_{2}\left(r_{2} / r_{1}, r_{B} / r_{1}\right)\right\}=0 .
$$

Equation (10) implies that

$$
v_{A}\left(r_{1}, r_{B}\right) \approx r_{1}^{\eta_{A}}
$$

Thus, $v_{A}$ is quite insensitive with respect to variation in $r_{B}$. (The more volatile the ratio $R / Z$ is, and the closer $r_{1}$ and $r_{B}$ are to 1 , the more $v_{A}\left(r_{1}, r_{B}\right)$ is sensitive with respect to variation in $r_{B}$.) We do not have equality in the limit when $r_{2} \rightarrow r_{1}$, since with probability zero, the time $T_{2}\left(r_{2} / r_{1}, r_{B} / r_{1}, \omega\right)$ is positive and in this time period we have that $R(s, \omega) / Z(s, \omega)>r_{2}$ and $R(s, \omega) / Z(s, \omega)$ may reach $r_{B}$ before $r_{2}$. In the approximation we neglect the possibility that $T_{2}$ does not vanish in the limit. By a similar argument, we have $v_{B}\left(r_{A}, r_{B}\right) \approx r_{B}^{\eta_{B}}$.

In the proof of Theorem 4.1 below, equation (19), it is shown that the optimal threshold satisfies

$$
v_{A}\left(m_{A}\left(r_{B}\right), r_{B}\right)=\left(\beta-\mu_{A, z}\right)\left(u_{A}\left(m_{A}\left(r_{B}\right), r_{B}\right)-\tau_{A}\right)
$$

if $u_{A}\left(m_{A}\left(r_{B}\right), r_{B}\right)>\tau_{A}$. Correspondingly, if $u_{B}\left(r_{A}, m_{B}\left(r_{A}\right)\right)>\tau_{B}$, then

$$
v_{B}\left(r_{A}, m_{B}\left(r_{A}\right)\right)=\left(\beta-\mu_{B, z}\right)\left(u_{B}\left(r_{A}, m_{B}\left(r_{A}\right)\right)-\tau_{B}\right) .
$$

When combining (11) and (12) we get the approximations

$$
\begin{aligned}
& u_{A}\left(m_{A}\left(r_{B}\right), r_{B}\right) \approx \frac{1}{\beta-\mu_{A, z}} m_{A}^{\eta_{A}}\left(r_{B}\right)+\tau_{A}, \\
& u_{B}\left(r_{A}, m_{B}\left(r_{A}\right)\right) \approx \frac{1}{\beta-\mu_{B, z}} m_{B}^{\eta_{B}}\left(r_{A}\right)+\tau_{B} .
\end{aligned}
$$

To obtain some intuition for these expressions, consider the case with time invariant adjustment outcome, $Z=1$, implying that $\mu_{\nu, z}=0$. If in addition, $\eta_{A}=1$ and $\eta_{B}=-1$, then (13) and (14) read $u_{A} \approx r_{A} / \beta+\tau_{A}$ and $u_{B} \approx 1 /\left(r_{B} \beta\right)+\tau_{B}$, which can be rearranged to $r_{A} \approx\left(u_{A}-\tau_{A}\right) \beta$ and $1 / r_{B} \approx\left(u_{B}-\tau_{B}\right) \beta$.

The following heuristic argument explains these expressions: By renegotiating the contract, a player incurs the adjustment fee, and then obtains the expected utility after an adjustment, $u_{\nu}$. Multiplying by the discount rate $\beta$, we obtain the equivalent flow payoff. A player should demand an adjustment when the real contract payment equals the equivalent flow payoff from requiring an adjustment, i.e., the critical thresholds are given by these formulas.

These approximations imply that the volatility only influences the thresholds through the expected objective functions $u_{\nu}$. These relations may be useful in order to find the optimal thresholds. Comparing with the numerical simulations in Section 4, these approximations underestimate $u_{A}$ and $u_{B}$ by about 2 percent. The approximation is better the smaller the volatility in $Z$ and $Q$.

\section{Appendix B. Proofs}

We have the following two technical results that are proved at the end of this section.

Lemma B.1. Assume the real adjustment outcome $Z$ and the aggregate price $Q$ are continuous and satisfy property $\mathcal{F}$. Then $v_{A}\left(r_{A}, r_{B}\right)$ is continuous, increasing in both variables and

$$
\frac{\partial v_{A}\left(r_{A}, r_{B}\right)}{\partial r_{B}}<\left(\beta-\mu_{A, z}\right) \frac{\partial u_{A}\left(r_{A}, r_{B}\right)}{\partial r_{B}} .
$$


Correspondingly, $v_{B}\left(r_{A}, r_{B}\right)$ is continuous, decreasing in both variables and

$$
\frac{\partial v_{B}\left(r_{A}, r_{B}\right)}{\partial r_{A}}>\left(\beta-\mu_{B, z}\right) \frac{\partial u_{B}\left(r_{A}, r_{B}\right)}{\partial r_{A}} .
$$

Lemma B.2. If the real adjustment outcome model $Z$ is continuous and satisfies property $\mathcal{F}$, then

$$
E\left\{1-Z^{\eta_{\nu}}(t) \exp (-\beta t)\right\}=\left(\beta-\mu_{\nu, z}\right) E\left\{\int_{0}^{t} Z^{\eta_{\nu}}(s) \exp (-\beta s) d s\right\} .
$$

Proof of Theorem 2.3. The objective function may be written

$$
\begin{aligned}
U_{\nu}\left(t_{1}, \ldots, \omega\right) & =\sum_{j=0}^{\infty}\left(\int_{t_{j}}^{t_{j+1}} R^{\eta_{\nu}}(s, \omega) \exp (-\beta s) d s-\tau_{\nu} Z^{\eta_{\nu}}\left(t_{j+1}, \omega\right) \exp \left(-\beta t_{j+1}\right)\right) \\
& =\tau_{\nu}+\sum_{j=0}^{\infty} Z^{\eta_{\nu}}\left(t_{j}, \omega\right) \exp \left(-\beta t_{j}\right)\left(\int_{t_{j}}^{t_{j+1}} \frac{Q^{\eta_{\nu}}\left(t_{j}, \omega\right)}{Q^{\eta_{\nu}}(s, \omega)} \exp \left(-\beta\left(s-t_{j}\right)\right) d s-\tau_{\nu}\right) .
\end{aligned}
$$

The expected value of integral in the last expression above is bounded due to property $\mathcal{F}$. Then $E\left\{U_{\nu}\right\}$ is bounded if the number of adjustments is finite.

If there is an infinite number of adjustments, it is in addition necessary to bound

$$
E\left\{\sum_{j=0}^{\infty} Z^{\eta_{\nu}}\left(t_{j}\right) \exp \left(-\beta t_{j}\right)\right\}
$$

This expression is bounded due to property $\mathcal{F}$.

When both $\tau_{A}, \tau_{B}>0$, neither player benefits from requiring adjustment immediately all the time, e.g., have a critical threshold equal to 1 . Hence the problem is well-defined.

Let $T$ satisfy $t_{i}<T \leq t_{i+1}$. Define $C_{T}$ as the contribution to the objective function for $t<T$ that cannot be changed when $t \geq T$, that is,

$$
\begin{aligned}
C_{T}=\sum_{j=0}^{i-1}\left(\int_{t_{j}}^{t_{j+1}} R^{\eta_{\nu}}(s, \omega) \exp (-\beta s) d s\right. & \left.-\tau_{\nu} Z^{\eta_{\nu}}\left(t_{j+1}, \omega\right) \exp \left(-\beta t_{j+1}\right)\right) \\
& +\int_{t_{i}}^{T} R^{\eta_{\nu}}(s, \omega) \exp (-\beta s) d s
\end{aligned}
$$

and $H_{\nu}\left(t_{i+2}-t_{i+1}, \ldots, \omega\right)$ as the contribution to the object function after $t_{i+1}$, that is,

$$
\begin{aligned}
H_{\nu}\left(t_{i+2}-t_{i+1}, \ldots, \omega\right)=\sum_{j=i+1}^{\infty}\left(\int_{t_{j}}^{t_{j+1}} R^{\eta_{\nu}}(s, \omega) \exp (-\beta s) d s\right. \\
\left.\quad-\tau_{\nu} Z^{\eta_{\nu}}\left(t_{j+1}, \omega\right) \exp \left(-\beta t_{j+1}\right)\right) .
\end{aligned}
$$

The function $H_{\nu}\left(t_{i+2}-t_{i+1}, \ldots, \omega\right)$ has the same distribution as $U_{\nu}\left(t_{0}, \ldots, \omega\right)$. Then we may write the objective function as

$$
\begin{aligned}
U_{\nu}\left(t_{1}, \ldots, \omega\right) & \\
=C_{T} & +Z^{\eta_{\nu}}(T, \omega) \exp (-\beta T)\left(\frac{R^{\eta_{\nu}}(T, \omega)}{Z^{\eta_{\nu}}(T, \omega)} \int_{T}^{t_{i+1}} \frac{R^{\eta_{\nu}}(s, \omega)}{R^{\eta_{\nu}}(T, \omega)} \exp (-\beta(s-T)) d s\right. \\
& \left.+\frac{Z^{\eta_{\nu}}\left(t_{i+1}, \omega\right)}{Z^{\eta_{\nu}}(T, \omega)} \exp \left(-\beta\left(t_{i+1}-T\right)\right)\left(H_{\nu}\left(t_{i+2}-t_{i+1}, \ldots, \omega\right)-\tau_{\nu}\right)\right) .
\end{aligned}
$$

The ratios $R(s, \omega) / R(T, \omega)$ and $Z\left(t_{i+1}, \omega\right) / Z(T, \omega)$ are independent of $R(T, \omega)$ and $Z(T, \omega)$ due to the Markov properties. The future contribution to the object function depends on $R(T, \omega)$ and $Z(T, \omega)$, but the optimal strategy is only a function of the ratio $R(T, \omega) / Z(T, \omega)$ and there is no memory in the game, i.e., dependencies on $t_{j}<T, R(s, \omega)$ for $s<T$ or $Z(s, \omega)$ for $s<T$.

Let $s_{B}$ and $s_{A}$ denote the strategies of player $\mathrm{B}$ and player A, respectively. With a slight abuse of notation, let $U_{A}\left(s_{A}, s_{B}, \omega\right)$ denote the objective function with the strategies $s_{A}$ and $s_{B}$, 
respectively. Then $\sup _{s_{A}} E\left\{U_{A}\left(s_{A}, s_{B}\right)\right\}$ is well-defined and there is a sequence $s_{A, i}$ such that

$$
\lim _{i \rightarrow \infty} E\left\{U_{A}\left(s_{A, i}, s_{B}\right)\right\}=\sup _{s_{A}} E\left\{U_{A}\left(s_{A}, s_{B}\right)\right\} .
$$

Define the sequence of sets $S_{i}$ where $r \in S_{i}$ if player A with strategy $s_{A, i}$ requires contract adjustment for any interval for any price $Q(\cdot, \omega)$ at time $t$ where $R(t, \omega) / Z(t, \omega)=r$. To ensure that the sets are non-empty, add the number 0 to $S_{i}$. If the adjustment is not the first time $t$ when $R(t, \omega) / Z(s, \omega)=r$, this is not critical, since it is the contribution to the objective function of the player in the future that is critical. Since all $r \in S_{i}$ satisfies $0 \leq r<1$, then for any sequence $\left\{r_{i}\right\}_{i}$ with $r_{i} \in S_{i}$, there is an accumulation point $r^{\prime}$ (if several, take the largest). Consider a strategy $s^{\prime}$ with a critical threshold $r^{\prime}$. Since the expected value of the future contribution to the objective function at time $t$ only is a function of the present $R(t, \omega) / Z(t, \omega)$, and equation (16), then

$$
E\left\{U_{A}\left(s^{\prime}, s_{B}\right)\right\}=\sup _{s_{A}} E\left\{U_{A}\left(s_{A}, s_{B}\right)\right\} .
$$

If the adjustment outcome $Z Q$ does not only change in discrete jumps, then the adjustments will come with shorter and shorter time intervals if $r_{A} \rightarrow 1$. Assuming $\tau_{A}>0$, then the adjustment cost dominates the objective function which implies that the accumulation point $r^{\prime}<1$. If the price $Z Q$ only changes in discrete jumps, then the relative flow payoff can only take discrete values and $r=1$ cannot be an accumulation point for the chain where all elements in the chain satisfies $r_{i}<0$. This implies that the critical threshold may be set equal to the accumulation point $0 \leq r^{\prime}<1$.

Correspondingly, if player A has the same strategy in each time interval, then there is a corresponding argument showing that there cannot be a better strategy for player B than what is possible to obtain with a critical threshold $r_{B}$.

Proof of Theorem 3.1. Let $H_{\nu}^{\prime}$ be defined as $H_{\nu}$ in the proof of Theorem 2.3 but with $T<t_{1}$ and with parameters $r_{A}$ and $r_{B}$ instead of $t_{1}-t_{0}, \ldots$ The definition of $U_{\nu}\left(r_{A}, r_{B}, \omega\right)$ in (1) and (4) implies

$$
U_{\nu}\left(r_{A}, r_{B}, \omega\right)=\int_{t_{0}}^{t_{1}} R^{\eta_{\nu}}(s, \omega) \exp (-\beta s) d s+Z^{\eta_{\nu}}\left(t_{1}, \omega\right) \exp \left(-\beta t_{1}\right)\left(H_{\nu}^{\prime}\left(r_{A}, r_{B}, \omega\right)-\tau_{\nu}\right) .
$$

The stochastic variables $U_{\nu}$ and $H_{\nu}^{\prime}$ have similar distribution and have expectation equal to $u_{\nu}$. The time for the end of the first interval $t_{1}$ is independent of what is happening after $t_{1}$ due to the Markov property. Hence $Z^{\eta_{\nu}}\left(t_{1}, \omega\right) \exp \left(-\beta t_{1}\right)$ is independent of $H_{\nu}^{\prime}\left(r_{A}, r_{B}, \omega\right)$. This implies that

$$
u_{\nu}\left(r_{A}, r_{B}\right)=f_{\nu}\left(r_{A}, r_{B}\right)-\tau_{\nu} h_{\nu}\left(r_{A}, r_{B}\right)+h_{\nu}\left(r_{A}, r_{B}\right) u_{\nu}\left(r_{A}, r_{B}\right)
$$

leading to

$$
u_{\nu}\left(r_{A}, r_{B}\right)=\frac{f_{\nu}\left(r_{A}, r_{B}\right)-\tau_{\nu} h_{\nu}\left(r_{A}, r_{B}\right)}{1-h_{\nu}\left(r_{A}, r_{B}\right)} .
$$

This equation may also be written as

$$
u_{\nu}\left(r_{A}, r_{B}\right)=\frac{f_{\nu}\left(r_{A}, r_{B}\right)-\tau_{\nu}}{1-h_{\nu}\left(r_{A}, r_{B}\right)}+\tau_{\nu}
$$

Assuming $f$ and $h$ are differentiable, the derivatives satisfy

$$
\frac{\partial u_{\nu}}{\partial r_{\mu}}=\frac{\frac{\partial f_{\nu}}{\partial r_{\mu}}\left(1-h_{\nu}\right)+\left(f_{\nu}-\tau_{\nu}\right) \frac{\partial h_{\nu}}{\partial r_{\mu}}}{\left(1-h_{\nu}\right)^{2}}=\frac{\frac{\partial f_{\nu}}{\partial r_{\mu}}+\left(u_{\nu}-\tau_{\nu}\right) \frac{\partial h_{\nu}}{\partial r_{\mu}}}{1-h_{\nu}}
$$

for $\mu=A, B$.

Proof of Theorem 4.1. (i) Using Lemma B.2 we have

$$
\frac{f_{A}}{1-h_{A}}=\frac{E\left\{\int_{0}^{T} R^{\eta_{A}}(s) \exp (-\beta s) d s\right\}}{\left(\beta-\mu_{A, z}\right) E\left\{\int_{0}^{T} Z^{\eta_{A}}(s) \exp (-\beta s) d s\right\}} .
$$


Hence the fraction on the left hand side is a constant times the average of $R^{\eta_{A}}$ until the first contract adjustment divided by the average of $Z^{\eta_{A}}$ in the same interval for $r_{A}<R / Z<r_{B}$. We will show that the fraction (18) is increasing in $r_{B}$. Let $r_{B}^{\prime}>r_{B}$ and

$$
T^{\prime}=\inf \left\{s \geq T \mid R(s, \omega) / Z(s, \omega) \notin\left(r_{A}, r_{B}^{\prime}\right)\right\} .
$$

Define further $T \leq T^{\prime \prime} \leq T^{\prime}$ as

$$
T^{\prime \prime}=\inf \left\{s \geq T \mid R(s, \omega) / Z(s, \omega) \notin\left(1, r_{B}^{\prime}\right)\right\} .
$$

The lower endpoint is set equal to 1 since $R(0, \omega) / Z(0, \omega)=1$. Then

$$
\begin{aligned}
E\left\{\int_{0}^{T^{\prime}} R^{\eta_{A}}(s) \exp (-\beta s) d s\right\}= & E\left\{\int_{0}^{T} R^{\eta_{A}}(s) \exp (-\beta s) d s\right\} \\
& +E\left\{\int_{T}^{T^{\prime \prime}} R^{\eta_{A}}(s) \exp (-\beta s) d s\right\} \\
& +E\left\{\int_{T^{\prime \prime}}^{T^{\prime}} R^{\eta_{A}}(s) \exp (-\beta s) d s\right\} .
\end{aligned}
$$

Let $P$ be the probability that there exists times $s_{1}$ and $s_{2}$ with $T<s_{1}<T^{\prime \prime}<s_{2}<T^{\prime}$ such that $R\left(s_{1}, \omega\right) / Z\left(s_{1}, \omega\right) \geq r_{B}$ and $R\left(s_{2}, \omega\right) / Z\left(s_{2}, \omega\right) \leq 1$. Then

$$
E\left\{\int_{T^{\prime \prime}}^{T^{\prime}} R^{\eta_{A}}(s) \exp (-\beta s) d s\right\}=P E\left\{\int_{0}^{T^{\prime}} R^{\eta_{A}}(s) \exp (-\beta s) d s\right\}
$$

since $R(\cdot, \omega) / Z(\cdot, \omega)$ varies in the same interval $\left(1, r_{B}^{\prime}\right)$ in both expressions. This implies that

Correspondingly, we have

$$
\begin{aligned}
E\left\{\int_{0}^{T^{\prime}} R^{\eta_{A}}(s) \exp (-\beta s) d s\right\}= & \frac{1}{1-P}\left(E\left\{\int_{0}^{T} R^{\eta_{A}}(s) \exp (-\beta s) d s\right\}\right. \\
& \left.+E\left\{\int_{T}^{T^{\prime \prime}} R^{\eta_{A}}(s) \exp (-\beta s) d s\right\}\right) .
\end{aligned}
$$

$$
\begin{aligned}
E\left\{\int_{0}^{T^{\prime}} Z^{\eta_{A}}(s) \exp (-\beta s) d s\right\}= & \frac{1}{1-P}\left(E\left\{\int_{0}^{T} Z^{\eta_{A}}(s) \exp (-\beta s) d s\right\}\right. \\
& \left.+E\left\{\int_{T}^{T^{\prime \prime}} Z^{\eta_{A}}(s) \exp (-\beta s) d s\right\}\right) .
\end{aligned}
$$

We have

$$
\frac{E\left\{\int_{T}^{T^{\prime \prime}} R^{\eta_{A}}(s) \exp (-\beta s) d s\right\}}{E\left\{\int_{T}^{T^{\prime \prime}} Z^{\eta_{A}}(s) \exp (-\beta s) d s\right\}}>\frac{E\left\{\int_{0}^{T} R^{\eta_{A}}(s) \exp (-\beta s) d s\right\}}{E\left\{\int_{0}^{T} Z^{\eta_{A}}(s) \exp (-\beta s) d s\right\}}
$$

since on the left-hand side $R(T, \omega) / Z(T, \omega) \geq r_{B}$, and $R(\cdot, \omega) / Z(\cdot, \omega)$ varies in the interval $\left(1, r_{B}^{\prime}\right)$ while on the right-hand side $R(0, \omega) / Z(0, \omega)=1$, and $R(\cdot, \omega) / Z(\cdot, \omega)$ varies in the interval $\left(r_{A}, r_{B}\right)$. Then

$$
\frac{E\left\{\int_{0}^{T^{\prime}} R^{\eta_{A}}(s) \exp (-\beta s) d s\right\}}{E\left\{\int_{0}^{T^{\prime}} Z^{\eta_{A}}(s) \exp (-\beta s) d s\right\}}>\frac{E\left\{\int_{0}^{T} R^{\eta_{A}}(s) \exp (-\beta s) d s\right\}}{E\left\{\int_{0}^{T} Z^{\eta_{A}}(s) \exp (-\beta s) d s\right\}} .
$$

Hence the fraction on the left hand side of (18) is increasing in $r_{B}$.

When $r_{B}$ increases, $E\{T\}$ increases and $h_{A}$ decreases due to property $\mathcal{F}$. This implies $-\tau_{A} /(1-$ $h$ ) increases when $r_{A}$ increases. Hence by using (17) we see that increasing $r_{B}$ increases $u_{A}$. The corresponding argument may be applied for $u_{B}\left(r_{A}, r_{B}\right)$.

(ii) Since $Z$ and $Q$ are continuous, $f_{A}, h_{A}$, and hence $u_{A}$ are continuous by Theorem 3.1. Then $u_{m, A}$ is continuous and $m_{A}$ is well-defined, piecewise continuous and $u_{A}\left(m_{A}\left(r_{B}\right), r_{B}\right)=u_{m, A}\left(r_{B}\right)$ and $u_{B}\left(r_{A}, m_{B}\left(r_{A}\right)\right)=u_{m, B}\left(r_{A}\right)$.

Let $r_{B}>1$ be fixed. We will first prove that there is a value $0 \leq r_{A}<1$ that maximizes $u_{A}\left(r_{A}, r_{B}\right)$. The function $u_{A}\left(r_{A}, r_{B}\right)$ is defined for $0 \leq r_{A}<1$. We will give an argument that the maximum value is attained for $r_{A}$ in the closed interval $[0,1-\epsilon]$ for $\epsilon>0$ sufficiently 
small. Since the interval is closed, the maximum value will be attained for a value $r_{A}=m_{A}\left(r_{B}\right)$. Since $Z$ and $Q$ are continuous, then $T\left(r_{A}, r_{B}\right)$ vanishes with probability 1 when $r_{A} \rightarrow 1$. Then $h_{A}\left(r_{A}, r_{B}\right) \rightarrow 1$ and $f_{A}\left(r_{A}, r_{B}\right) \rightarrow 0$ when $r_{A} \rightarrow 1$. Further, the expression (5) for $u_{A}$ implies that $u_{A}\left(r_{A}, r_{B}\right) \rightarrow-\infty$ when $r_{A} \rightarrow 1$. Hence for each value for $r_{B}$, there is a value $r_{A}=m_{A}\left(r_{B}\right)<1$ where $u_{A}\left(r_{A}, r_{B}\right)=u_{m, A}\left(r_{B}\right)$.

We will prove that the value $r_{A}=m_{A}\left(r_{B}\right)$ is unique, i.e., if $r_{A} \neq m_{A}\left(r_{B}\right)$, then $u_{A}\left(r_{A}, r_{B}\right)<$ $u_{m, A}\left(r_{B}\right)$. Let $t_{1}$ be the time for the first contract adjustment. Furthermore, let $W_{1}$ and $W_{2}$ be two new stochastic variables that are identical to $U_{A}$ except that a different strategy (a different limit for $r_{A}$ ) is used before $t_{1}$. After $t_{1}$, we set $r_{A}=m_{A}\left(r_{B}\right)$. The first contract adjustment in $W_{2}$ and $W_{1}$, if required by player $\mathrm{A}$, is required when $R / Z$ reaches the values $r_{2}<r_{1}<1$, respectively. Let $P_{r_{1}}$ denote the probability that $R(t, \omega) / Z(t, \omega)$ reaches $r_{1}$ before it reaches $r_{B}$. In case the ratio reaches $r_{B}$ first, there is no difference between $W_{1}$ and $W_{2}$. Further, let $T_{1}=T\left(r_{1}, r_{B}, \omega\right)$ denote the time of the first contract adjustment for $W_{1}$. Finally, let

$$
E_{r_{1}}=E\left\{\exp \left(-\beta T_{1}\left(r_{1}, r_{B}\right)\right)\right\}
$$

given that $r_{1}$ is reached. For the strategy associated with $W_{2}$ there is a contract adjustment when $R / Z$ reaches $r_{2}$ or $r_{B}$. Assuming $r_{1}$ is reached, there is contract adjustment when either the ratio decreases with a factor $r_{2} / r_{1}$ or increases with a factor $r_{B} / r_{1}$. Let $T_{2}=T\left(r_{2} / r_{1}, r_{B} / r_{1}, \omega\right)$ denote the time between $r_{1}$ is reached and either $r_{2}$ or $r_{B}$ is reached. Let $w_{1}$ and $w_{2}$ be the expected values of $W_{1}$ and $W_{2}$, respectively. Assuming that $r_{1}$ is reached before $r_{B}$, then

$$
w_{1}=E\left\{C_{T_{1}}\right\}+E_{r_{1}} E\left\{\left(u_{m, A}\left(r_{B}\right)-\tau_{A}\right) Z^{\eta_{A}}\left(T_{1}\right) \exp \left(-\beta T_{2}\right)\right\}
$$

and

$$
\begin{array}{rl}
w_{2}=E\left\{C_{T_{1}}\right\}+E_{r_{1}} & E\left\{\int_{0}^{T_{2}} R^{\eta_{A}}\left(T_{1}+s\right) \exp (-\beta s) d s\right. \\
& \left.+\left(u_{m, A}\left(r_{B}\right)-\tau_{A}\right) Z^{\eta_{A}}\left(T_{1}+T_{2}\right) \exp \left(-\beta T_{2}\right)\right\}
\end{array}
$$

where $C_{T_{1}}$ is defined in (15). As noted above, $W_{1}=W_{2}$ are identical except if $r_{1}$ is reached. Since the probability that $r_{1}$ is reached before $r_{B}$ is $P_{r_{1}}$, the difference is

$$
\begin{aligned}
& w_{2}-w_{1}=P_{r_{1}} E_{r_{1}} E\left\{\int_{0}^{T_{2}} R^{\eta_{A}}\left(T_{1}+s\right) \exp (-\beta s) d s\right. \\
&\left.\quad-\left(u_{m, A}\left(r_{B}\right)-\tau_{A}\right)\left(Z^{\eta_{A}}\left(T_{1}\right)-Z^{\eta_{A}}\left(T_{1}+T_{2}\right) \exp \left(-\beta T_{2}\right)\right)\right\} \\
&=P_{r_{1}} E_{r_{1}} E\left\{\int_{0}^{T_{2}} R^{\eta_{A}}\left(T_{1}+s\right) \exp (-\beta s) d s\right. \\
&\left.\quad-\left(\beta-\mu_{A, z}\right)\left(u_{m, A}\left(r_{B}\right)-\tau_{A}\right) \int_{0}^{T_{2}} Z^{\eta_{A}}\left(T_{1}+s\right) \exp (-\beta s) d s\right\} \\
&=P_{r_{1}} E_{r_{1}}\left(\frac{E\left\{\int_{0}^{T_{2}} R^{\eta_{A}}\left(T_{1}+s\right) \exp (-\beta s) d s\right\}}{E\left\{\int_{0}^{T_{2}} Z^{\eta_{A}}\left(T_{1}+s\right) \exp (-\beta s) d s\right\}}-\left(\beta-\mu_{A, z}\right)\left(u_{m, A}\left(r_{B}\right)-\tau_{A}\right)\right) \\
& \times E\left\{\int_{0}^{T_{2}} Z^{\eta_{A}}\left(T_{1}+s\right) \exp (-\beta s) d s\right\} .
\end{aligned}
$$

Lemma B.2 is used in the second equality. Define the limit of the discounted real adjustment outcome when the lower threshold is slightly reduced by

$$
L_{A}\left(r_{1}, r_{B}\right)=\lim _{r_{2} \rightarrow r_{1}-} \frac{E\left\{\int_{0}^{T_{2}} Z^{\eta_{A}}\left(T_{1}+s\right) \exp (-\beta s) d s\right\}}{r_{2}-r_{1}} .
$$

Due to the Markov property and property $\mathcal{F}$, the numerator is monotone and hence the limit is well-defined. Letting $r_{2} \rightarrow r_{1}$, we have

$$
\frac{\partial w_{1}}{\partial r_{1}}=-P_{r_{1}} E_{r_{1}}\left(v_{A}\left(r_{1}, r_{B}\right)-\left(\beta-\mu_{A, z}\right)\left(u_{m, A}\left(r_{B}\right)-\tau_{A}\right)\right) L_{A}\left(r_{1}, r_{B}\right)
$$


where $v_{A}$ is defined by (9). Hence, $\frac{\partial w_{1}}{\partial r_{1}}=0$ when

$$
v_{A}\left(r_{1}, r_{B}\right)=\left(\beta-\mu_{A, z}\right)\left(u_{m, A}\left(r_{B}\right)-\tau_{A}\right) .
$$

According to Theorem 3.1 and Lemma B.1, $u_{m, A}\left(r_{B}\right)$ and $v_{A}\left(r_{1}, r_{B}\right)$ are continuous and $v_{A}\left(r_{1}, r_{B}\right)$ is increasing in $r_{1}$. Consider the function $w_{1}\left(r_{1}, r_{B}\right)$ with $r_{B}$ fixed. Then $w_{1}\left(r_{1}, r_{B}\right)$ reaches its maximum with respect to $r_{1}$ either for $r_{1}=0$ or for a value $r_{1}>0$ when $\frac{\partial w_{1}\left(r_{1}, r_{B}\right)}{\partial r_{1}}=0$. Since $v_{A}\left(r_{1}, r_{B}\right)$ is increasing in $r_{1}$, then $\frac{\partial w_{1}}{\partial r_{1}}$ changes sign when (19) is satisfied. Hence, the maximum is unique. Since the periods between contract adjustments are independent then the value $r_{1}$ that maximizes $w_{1}$ also maximizes $u_{A}\left(r_{A}, r_{B}\right)$. Hence, there is a unique value $r_{A}=m_{A}\left(r_{B}\right)$ that maximizes $u_{A}\left(r_{A}, r_{B}\right)$.

The corresponding argument may be applied for $u_{B}\left(r_{A}, r_{B}\right)$. However, since $r_{B}$ varies in an unbounded interval we should consider $u_{B}$ as a function of $1 / r_{B}$ instead of $r_{B}$ when applying the argument. This is possible since $u_{B}\left(r_{A}, r_{B}\right)$ is well-defined as $r_{B}$ approaches $\infty$.

(iii) Above it is proved that the optimal value $m_{A}\left(r_{B}\right)$ satisfies equation

$$
v_{A}\left(m_{A}\left(r_{B}\right), r_{B}\right)=\left(\beta-\mu_{A, z}\right)\left(u_{A}\left(m_{A}\left(r_{B}\right), r_{B}\right)-\tau_{A}\right) .
$$

Differentiating both sides with respect to $r_{B}$ gives

$$
\frac{\partial v_{A}}{\partial r_{A}} \frac{d m_{A}}{d r_{B}}+\frac{\partial v_{A}}{\partial r_{B}}=\left(\beta-\mu_{A, z}\right)\left(\frac{\partial u_{A}}{\partial r_{A}} \frac{d m_{A}}{d r_{B}}+\frac{\partial u_{A}}{\partial r_{B}}\right) .
$$

Since

$$
\frac{\partial v_{A}}{\partial r_{B}}<\left(\beta-\mu_{A, z}\right) \frac{\partial u_{A}}{\partial r_{B}}
$$

from Lemma B.1 and $\partial u_{A} / \partial r_{A}=0$ since $m_{A}\left(r_{B}\right)$ is the optimal value of $r_{A}$, this implies that

$$
\frac{\partial v_{A}}{\partial r_{A}} \frac{d m_{A}}{d r_{B}}>0
$$

Since $\partial v_{A} / \partial r_{A}>0$, then also $d m_{A} / d r_{B}>0$, i.e., $m_{A}\left(r_{B}\right)$ is a strictly increasing function. The proof that $m_{B}\left(r_{A}\right)$ is strictly increasing is similar.

(iv) Equation (6) may be used in order to prove that $m_{A}\left(r_{B}\right)$ decreases when $\tau_{A}$ increases, assuming $m_{A}\left(r_{B}\right)>0$. The function $u_{A}\left(r_{A}, r_{B}\right)$ has an optimal value for $r_{A}=m_{A}\left(r_{B}\right)>0$. Since $u_{A}$ is differentiable, there exists an $\varepsilon>0$ such that $\frac{\partial u_{A}}{\partial r_{A}}\left(r_{A}, r_{B}\right)>0$ for $m_{A}\left(r_{B}\right)-\varepsilon<r_{A}<m_{A}\left(r_{B}\right)$ and $\frac{\partial u_{A}}{\partial r_{A}}\left(r_{A}, r_{B}\right)<0$ for $m_{A}\left(r_{B}\right)<r_{A}<m_{A}\left(r_{B}\right)+\varepsilon$. If $\tau_{A}$ is increased, then $\frac{\partial u_{A}}{\partial r_{A}}$ is decreased which implies a reduction in the $r_{A}$ value where $\frac{\partial u_{A}}{\partial r_{A}}=0$. This implies that increasing the adjustment fee reduces the optimal threshold value $m_{A}\left(r_{B}\right)$. Correspondingly, it is proved that $m_{B}\left(r_{A}\right)$ strictly increases when $\tau_{B}$ increases assuming $m_{B}\left(r_{A}\right)>0$.

(v) Since $u_{m, \nu}$ and $v_{\nu}$ are continuous, we infer that the functions $m_{A}\left(r_{B}\right):(1, \infty] \rightarrow[0,1)$ and $m_{B}\left(r_{A}\right):[0,1) \rightarrow(1, \infty]$ are continuous. In the infinite rectangle defined by $0 \leq r_{A}<1$ and $r_{B}>1, m_{A}\left(r_{B}\right)$ gives a continuous path between the lines defined by $r_{B}=0$ and $r_{B}=\infty$. Similarly, $m_{B}\left(r_{A}\right)$ gives a path in the same rectangle between the lines defined by $r_{A}=0$ and $r_{A}=1$. Hence, these two curves must intersect at least once, giving an equilibrium point.

Proof of Theorem 5.2. The existence of at least one equilibrium point $\left(r_{A}^{e}, r_{B}^{e}\right)$ is proved similarly as in Theorem 4.1 where it is assumed that the price $Z Q$ is continuous, i.e., the equilibrium point is the intersection between $m_{A}\left(r_{B}\right)$ and $m_{B}\left(r_{A}\right)$. But in this case, these curves are not necessarily continuous which implies that there might not be an intersection.

Define graphs $M_{A}$ and $M_{B}$ by extending the curves $m_{A}\left(r_{B}\right)$ and $m_{B}\left(r_{A}\right)$ by continuity as follows: Wherever $m_{A}\left(r_{B}\right)$ or $m_{B}\left(r_{A}\right)$ make jumps, connect the two sides across the jump by straight lines with constant $r_{B}$ and $r_{A}$, respectively. (See Figure 7.) Since $M_{A}$ and $M_{B}$ are continuous, they must intersect. If the intersection is on the straight lines, then randomization is necessary as illustrated in Section 5 . Assume $m_{B}\left(r_{A}\right)$ intersects a straight line in $M_{A}$ connecting the two points $\left(r_{1}, r_{B}^{e}\right)$ and $\left(r_{2}, r_{B}^{e}\right)$. Then $m_{B}\left(r_{1}\right)$ and $m_{B}\left(r_{2}\right)$ give values of $r_{B}$ on opposite side of $r_{B}^{e}$. We may then define a one parameter family of strategies $s_{A}$ where the probability for choosing $r_{1}$ varies in the interval $0 \leq q \leq 1$. Since $m_{B}\left(r_{1}\right)$ and $m_{B}\left(r_{2}\right)$ give values of $r_{B}$ on opposite side of $r_{B}^{e}$, then also the endpoints $m_{B}^{c}\left(s_{A}\right)$ when $s_{A}$ varies in the one-parameter family give values 
on the opposite side of $r_{B}^{e}$. The continuity of $m_{B}^{c}\left(s_{A}\right)$ ensures that there is a strategy $s_{A}^{e}$ that randomizes $r_{A}$ between $r_{1}$ and $r_{2}$ such that $m_{B}^{c}\left(s_{A}^{e}\right)=r_{B}$. There is a corresponding argument if $m_{A}\left(r_{B}\right)$ intersects a straight line in $M_{B}$.

The continuity of $m_{A}^{c}\left(s_{B}\right)$ and $m_{B}^{c}\left(s_{A}\right)$ implies that it is not necessary that both players randomize at the same time. If $M_{A}$ and $M_{B}$ intersect with two straight lines, then there may be two Nash equilibria defined by using $m_{A}^{c}\left(s_{B}\right)$ and $m_{B}^{c}\left(s_{A}\right)$, respectively.

Proof of Lemma B.1. Assume $R\left(T_{1}, \omega\right) / Z\left(T_{1}, \omega\right)=r_{1}$. Define $T_{3}\left(c, r_{B} / r_{1}\right)$ as the first time after $T_{1}$ where either $R\left(T_{1}+T_{3}, \omega\right) / Z\left(T_{1}+T_{3}, \omega\right)=c r_{1}$ or $R\left(T_{1}+T_{3}, \omega\right) / Z\left(T_{1}+T_{3}, \omega\right)=r_{B}$ for a constant $0<c<1$. Define

$$
v_{A}^{c}\left(r_{1}, r_{B}\right)=\frac{E\left\{\int_{0}^{T_{3}} R^{\eta_{A}}\left(T_{1}+s\right) \exp (-\beta s) d s\right\}}{E\left\{\int_{0}^{T_{3}} Z^{\eta_{A}}\left(T_{1}+s\right) \exp (-\beta s) d s\right\}},
$$

i.e., $\lim _{c \rightarrow 1-} v_{A}^{c}\left(r_{1}, r_{B}\right)=v_{A}\left(r_{1}, r_{B}\right)$. Let $r_{2}<c r_{1}$ and consider the function $v_{A}^{c}\left(r_{2}, r_{B}\right)$. Assume $R\left(T_{2}, \omega\right) / Z\left(T_{2}, \omega\right)=r_{2}$ and define $T_{4}\left(c, r_{B} / r_{2}\right)$ as the first time after $T_{2}$ where either $R\left(T_{2}+\right.$ $\left.T_{4}, \omega\right) / Z\left(T_{2}+T_{4}, \omega\right)=c r_{2}$ or $R\left(T_{2}+T_{4}, \omega\right) / Z\left(T_{2}+T_{4}, \omega\right)=r_{B}$. The interval $\left(T_{2}, T_{2}+T_{4}\right)$ may consist of several intervals $\left(t_{i}^{1}, t_{i}^{2}\right)$, i.e., $T_{2}<t_{i}^{1}<t_{i}^{2} \leq T_{2}+T_{4}$ that satisfies the properties of a $\left(T_{1}, T_{1}+T_{3}\right)$ interval, i.e., $R\left(t_{i}^{1}, \omega\right) / Z\left(t_{i}^{1}, \omega\right)=r_{1}, c r_{1}<R(s, \omega) / Z(s, \omega)<r_{B}$ for $t_{i}^{1}<s<t_{i}^{2}$ and $R\left(t_{i}^{2}, \omega\right) / Z\left(t_{i}^{2}, \omega\right)=c r_{1}$ or $R\left(t_{i}^{2}, \omega\right) / Z\left(t_{i}^{2}, \omega\right)=r_{B}$. Let $\Omega_{1}=\cup_{i}\left(t_{i}^{1}, t_{i}^{2}\right)$ and $\Omega_{2}=\left(T_{2}, T_{2}+T_{4}\right) \backslash \Omega_{1}$. Then

$$
\begin{aligned}
& v_{A}^{c}\left(r_{2}, r_{B}\right) \\
& \quad=\frac{E\left\{\int_{\Omega_{1}} R^{\eta_{A}}\left(T_{1}+s\right) \exp (-\beta s) d s\right\}+E\left\{\int_{\Omega_{2}} R^{\eta_{A}}\left(T_{1}+s\right) \exp (-\beta s) d s\right\}}{E\left\{\int_{\Omega_{1}} Z^{\eta_{A}}\left(T_{1}+s\right) \exp (-\beta s) d s\right\}+E\left\{\int_{\Omega_{2}} Z^{\eta_{A}}\left(T_{1}+s\right) \exp (-\beta s) d s\right\}} .
\end{aligned}
$$

We have that

$$
v_{A}^{c}\left(r_{1}, r_{B}\right)=\frac{E\left\{\int_{\Omega_{1}} R^{\eta_{A}}\left(T_{1}+s\right) \exp (-\beta s) d s\right\}}{E\left\{\int_{\Omega_{1}} Z^{\eta_{A}}\left(T_{1}+s\right) \exp (-\beta s) d s\right\}},
$$

since each interval $\left(t_{i}^{1}, t_{i}^{2}\right)$ has the same properties as $\left(T_{1}, T_{1}+T_{3}\right)$. Furthermore, we have that

$$
E\left\{\int_{\Omega_{2}} R^{\eta_{A}}\left(T_{1}+s\right) \exp (-\beta s) d s\right\}=\gamma_{c} E\left\{\int_{\Omega_{2}} Z^{\eta_{A}}\left(T_{1}+s\right) \exp (-\beta s) d s\right\}
$$

for a value $\left(c r_{2}\right)^{\eta_{A}}<\gamma_{c}<r_{1}^{\eta_{A}}$ since $\gamma_{c}$ is a weighted average of $R^{\eta_{A}}\left(T_{1}+s, \omega\right)$ divided by the weighted average of $Z^{\eta_{A}}\left(T_{1}+s, \omega\right)$ where the ratio $R / Z$ is varying in the same interval for each $\omega$ and pointwise in the integral. This implies that

$$
\begin{aligned}
v_{A}^{c}\left(r_{2}, r_{B}\right)= & v_{A}^{c}\left(r_{1}, r_{B}\right) E\left\{\int_{\Omega_{1}} Z^{\eta_{A}}\left(T_{1}+s\right) \exp (-\beta s) d s\right\} \\
& \left.+\gamma_{c} E\left\{\int_{\Omega_{2}} Z^{\eta_{A}}\left(T_{1}+s\right) \exp (-\beta s) d s\right\}\right] \\
\times & {\left[E\left\{\int_{\Omega_{1}} Z^{\eta_{A}}\left(T_{1}+s\right) \exp (-\beta s) d s\right\}\right.} \\
& \left.+E\left\{\int_{\Omega_{2}} Z^{\eta_{A}}\left(T_{1}+s\right) \exp (-\beta s) d s\right\}\right]^{-1} \\
= & (1-d) v_{A}^{c}\left(r_{1}, r_{B}\right)+d \gamma_{c},
\end{aligned}
$$

for $0<d<1$. Note that $\gamma_{c}<r_{1}^{\eta_{A}}$ and $d>0$ also when $c \rightarrow 0$ since $\Omega_{2}$ cannot be empty due to the first interval $0<s<t_{1}^{1}$ where $c r_{2}<R\left(T_{1}+s, \omega\right) / Z\left(T_{1}+s, \omega\right)<r_{1}$. This implies that $v_{A}^{c}\left(r_{2}, r_{B}\right)<v_{A}^{c}\left(r_{1}, r_{B}\right)$. Since $\lim _{c \rightarrow 1-} v_{A}^{c}\left(r_{1}, r_{B}\right)=v_{A}\left(r_{1}, r_{B}\right)$, then $v_{A}\left(r_{1}, r_{B}\right)$ is increasing in $r_{1}$. The function $v_{A}\left(r_{1}, r_{B}\right)$ is increasing and continuous in $r_{B}$ since this makes it possible with high $R(t, \omega) / Z(t, \omega)$ values and the probability for reaching $r_{B}$ changes continuously. From the definition of $v_{A}$ we have that $c v_{A}\left(r_{1}, r_{B}\right)=v_{A}\left(c r_{1}, c r_{B}\right)$. This implies that $v_{A}\left(r_{1}, r_{B}\right)$ also is continuous in $r_{1}$. The function $v_{B}\left(r_{A}, r_{B}\right)$ has similar properties. This function is decreasing in both arguments since $\eta_{B}<0$. 
The proof that

$$
\frac{\partial v_{A}}{\partial r_{B}}<\left(\beta-\mu_{A, z}\right) \frac{\partial u_{A}}{\partial r_{B}}
$$

is quite similar to the above argument. Assume $R\left(T_{5}, \omega\right) / Z\left(T_{5}, \omega\right)=r_{1} / c$ where $0<c<1$. Define $T_{6}\left(c, r_{B} /\left(c r_{1}\right)\right)$ as the first time after $T_{5}$ where either $R\left(T_{5}+T_{6}, \omega\right) / Z\left(T_{5}+T_{6}, \omega\right)=r_{1}$ or $R\left(T_{5}+T_{6}, \omega\right) / Z\left(T_{5}+T_{6}, \omega\right)=r_{B}$. Consider $v_{A}^{c}\left(r_{1} / c, r_{B}\right)$ and compare this with $u_{A}\left(r_{1}, r_{B}\right)$ where $R(s, \omega) / Z(s, \omega)$ starts at 1 and ends at either $r_{1}$ or $r_{B}$. Define $T_{7}>0$ as the first time $R\left(T_{5}+T_{7}, \omega\right) / Z\left(T_{5}+T_{7}, \omega\right)=1$, if this value is obtained. Define $\Omega_{1}=\left(T_{7}, T_{6}\right)$ and $\Omega_{2}=$ $\left(T_{5}, T_{6}\right) \backslash \Omega_{1}$. Note that $\Omega_{1}$ may be empty. Then

$$
\begin{aligned}
& v_{A}^{c}\left(r_{1} / c, r_{B}\right) \\
& \quad=\frac{E\left\{\int_{\Omega_{1}} R^{\eta_{A}}\left(T_{5}+s\right) \exp (-\beta s) d s\right\}+E\left\{\int_{\Omega_{2}} R^{\eta_{A}}\left(T_{5}+s\right) \exp (-\beta s) d s\right\}}{E\left\{\int_{\Omega_{1}} Z^{\eta_{A}}\left(T_{5}+s\right) \exp (-\beta s) d s\right\}+E\left\{\int_{\Omega_{2}} Z^{\eta_{A}}\left(T_{5}+s\right) \exp (-\beta s) d s\right\}} .
\end{aligned}
$$

Using (5) assuming for a moment that $\tau_{A}=0$ and using Lemma B.2 gives

$$
\left(\beta-\mu_{A, z}\right) \frac{f_{A}}{1-h_{A}}=\frac{E\left\{\int_{\Omega_{1}} R^{\eta_{A}}\left(T_{5}+s\right) \exp (-\beta s) d s\right\}}{E\left\{\int_{\Omega_{1}} Z^{\eta_{A}}\left(T_{5}+s\right) \exp (-\beta s) d s\right\}},
$$

since the interval $\left(T_{7}, T_{6}\right)$ has the same properties as in the variation of $u_{A}$. Furthermore, we have that

$$
E\left\{\int_{\Omega_{2}} R^{\eta_{A}}\left(T_{5}+s\right) \exp (-\beta s) d s\right\}=\gamma_{c} E\left\{\int_{\Omega_{2}} Z^{\eta_{A}}\left(T_{5}+s\right) \exp (-\beta s) d s\right\}
$$

for a value $r_{2}^{\eta_{A}}<\gamma_{c}<1$ by the same argument as above since in this case $r_{2}<R / Z<1$. This implies that

$$
\begin{aligned}
v_{A}^{c}\left(r_{2}, r_{B}\right)= & \left(\beta-\mu_{A, z}\right) \frac{f_{A}}{1-h_{A}} E\left\{\int_{\Omega_{1}} Z^{\eta_{A}}\left(T_{5}+s\right) \exp (-\beta s) d s\right\} \\
& \left.+\gamma_{c} E\left\{\int_{\Omega_{2}} Z^{\eta_{A}}\left(T_{5}+s\right) \exp (-\beta s) d s\right\}\right] \\
\times & {\left[E\left\{\int_{\Omega_{1}} Z^{\eta_{A}}\left(T_{5}+s\right) \exp (-\beta s) d s\right\}\right.} \\
& \left.+E\left\{\int_{\Omega_{2}} Z^{\eta_{A}}\left(T_{5}+s\right) \exp (-\beta s) d s\right\}\right]^{-1} \\
= & (1-d)\left(\beta-\mu_{A, z}\right) \frac{f_{A}}{1-h_{A}}+d \gamma_{c},
\end{aligned}
$$

for $0<d<1$. Note that $\gamma_{c}$ is independent of $r_{B}$ and $d>0$ since $\Omega_{2}$ cannot be empty due to the first interval $0<s<T_{7}$. Since $\lim _{c \rightarrow 1-} v_{A}^{c}\left(r_{1} / c, r_{B}\right)=v_{A}\left(r_{1}, r_{B}\right)$ and $\partial h_{A} / \partial r_{B}<0$, we have

$$
\frac{\partial v_{A}}{\partial r_{B}}<\left(\beta-\mu_{A, z}\right) \frac{\partial}{\partial r_{B}}\left(\frac{f_{A}}{1-h_{A}}\right)<\left(\beta-\mu_{A, z}\right) \frac{\partial}{\partial r_{B}}\left(\frac{f_{A}-\tau_{A}}{1-h_{A}}\right)=\left(\beta-\mu_{A, z}\right) \frac{\partial u_{A}}{\partial r_{B}} .
$$

Correspondingly, it is proved that

$$
\frac{\partial v_{B}}{\partial r_{A}}>\left(\beta-\mu_{B, Z}\right) \frac{\partial u_{B}}{\partial r_{A}}
$$

Note that since $\eta_{B}<0$ both expressions above are negative.

Proof of Lemma B.2. Let $t_{i}=i t / n$ and $Z(0, \omega)=1$. Then

$$
\begin{aligned}
E\left\{1-Z^{\eta_{\nu}}(t) \exp (-\beta t)\right\} & \\
= & E\left\{\sum_{i=0}^{n}\left(Z^{\eta_{\nu}}\left(t_{i}\right) \exp \left(-\beta t_{i}\right)-Z^{\eta_{\nu}}\left(t_{i+1}\right) \exp \left(-\beta t_{i+1}\right)\right)\right\} \\
& =E\left\{\sum_{i=0}^{n} Z^{\eta_{\nu}}\left(t_{i}\right) \exp \left(-\beta t_{i}\right)\left(1-\frac{Z^{\eta_{\nu}}\left(t_{i+1}\right)}{Z^{\eta_{\nu}}\left(t_{i}\right)} \exp \left(-\beta\left(t_{i+1}-t_{i}\right)\right)\right\}\right.
\end{aligned}
$$




$$
=\frac{1}{t_{1}} E\left\{1-Z^{\eta_{\nu}}\left(t_{1}\right) \exp \left(-\beta t_{1}\right)\right\} E\left\{\sum_{i=0}^{n} Z^{\eta_{\nu}}\left(t_{i}\right) \exp \left(-\beta t_{i}\right) t_{1}\right\}
$$

We have that

$$
\begin{aligned}
\lim _{t \rightarrow 0} E\left\{\frac{1-Z^{\eta_{\nu}}(t) \exp (-\beta t)}{t}\right\} & =\lim _{t \rightarrow 0} \frac{1-E\left\{Z^{\eta_{\nu}}(t)\right\} \exp (-\beta t)}{t} \\
& =\lim _{t \rightarrow 0} \frac{1-\exp \left(t\left(\mu_{\nu, z}-\beta\right)\right)}{t} \\
& =\beta-\mu_{\nu, z}
\end{aligned}
$$

and

$$
\lim _{n \rightarrow \infty} E\left\{\sum_{i=0}^{n} Z^{\eta_{\nu}}\left(t_{i}\right) \exp \left(-\beta t_{i}\right) t_{1}\right\}=E\left\{\int_{0}^{t} Z^{\eta_{\nu}}(s) \exp (-\beta s) d s\right\}
$$

Combining these three calculations proves the lemma.

Acknowledgment We are grateful to Karl Ove Moene and participants at a seminar at the University of Oslo for valuable comments to an earlier draft, and to Geir Asheim, Fred Espen Benth, and Bernt Øksendal for helpful discussions.

\section{REFERENCES}

[1] P. Aghion, M. Dewatripont, and P. Rey. Renegotiation design with unverifiable information. Econometrica 62, (1994) 257-282.

[2] T. Andersen and M. Stampe Christensen. Contract renegotiation under uncertainty. Working paper No 1997-5, Dep. of Economics, School of Economics and Management, University of Aarhus.

[3] T. Andersen and M. Stampe Christensen. Contract renewal under uncertainty. J. Economic Dynamics ES Control 26, (2002) 637-652.

[4] A. N. Borodin and P. Salminen. Handbook of Brownian Motion — Facts and Formulae. Birkhäuser, Basel (1996).

[5] A. Caplin and J. Leahy. Aggregation and optimization with state-dependent pricing. Econometrica 65, (1997) 601-625.

[6] A. Caplin and D. F. Spulber. Menu costs and the neutrality of money. Quarterly Journal of Economics CII, (1987) 703-725.

[7] L. Danziger. Price adjustment with stochastic inflation. International Economic Review 24, (1983) 699-707.

[8] L. Danziger. Contract Reopeners. Journal of Labor Economics 13, (1995) 62-87.

[9] L. Danziger. Delays in Renewal on Labor Contracts. Theory and Evidence. IZA Discussion Paper 709, (2003).

[10] E. Dockner, S. Jørgensen, N. Van Long, and G. Sorger. Differential Games in Economics and Management Science. Cambridge University Press, Cambridge (2000).

[11] P. Grout. Investment and wages in the absence of binding contracts. A Nash bargaining approach. Econometrica 52, (1984) 449-460.

[12] H. Haller and S. Holden Ratification Requirement and Bargaining Power. International Economic Review 38, (1998) 825-851.

[13] O. Hart and J. Moore. Incomplete contracts and renegotiation. Econometrica 56, (1988) 755-785.

[14] S. Holden. Wage bargaining and nominal rigidities. European Economic Review 38, (1994) $1021-1039$.

[15] S. Holden. Wage bargaining, holdouts and inflation. Oxford Economic Papers 49, (1997) 235-255.

[16] S. Holden. Renegotiation and the efficiency of investments. Rand Journal of Economics 30, (1999) 106-119.

[17] R. Isaacs. Differential Games. Wiley, New York (1965).

[18] D. E. Lebow, R. E. Saks, and B. A. Wilson. Downward nominal wage rigidity: Evidence from the Employment cost index. Advances in Macroeconomics 3, (2003), Issue 1, Article 2.

[19] W. B. MacLeod, and J. M. Malcomson. Investment, holdup, and the form of market contracts. American Economic Review 37, (1993) 343-354.

[20] J. M. Malcomson. Contracts, holdup and labor markets. Journal of Economic Literature 35, (1997) $1916-1957$.

[21] M. J. Osborne and A. Rubinstein. Bargaining and Markets. San Diego, Academic Press (1990).

[22] K.-I. Sato. Lévy Processes and Infinitely Divisible Distributions. Cambridge University Press, Cambridge (1999).

[23] E. Sheshinski and Y. Weiss. Inflation and costs of price adjustment. Review of Economic Studies 50, (1983) $513-529$.

[24] D. E. Spencer. The relative stickiness of wages and prices. Economic Inquiry 36, (1998) 120-137. 


\title{
CESifo Working Paper Series
}

\author{
(for full list see www.cesifo-group.de)
}

1409 Christian Gollier, Some Aspects of the Economics of Catastrophe Risk Insurance, February 2005

1410 Gebhard Kirchgässner, The Weak Rationality Principle in Economics, February 2005

1411 Carlos José Fonseca Marinheiro, Has the Stability and Growth Pact Stabilised? Evidence from a Panel of 12 European Countries and Some Implications for the Reform of the Pact, February 2005

1412 Petter Osmundsen, Frank Asche, Bård Misund and Klaus Mohn, Valuation of International Oil Companies -The RoACE Era, February 2005

1413 Gil S. Epstein and Shmuel Nitzan, Lobbying and Compromise, February 2005

1414 Marcel F. M. Canoy, Jan C. van Ours and Frederick van der Ploeg, The Economics of Books, February 2005

1415 Eric A. Hanushek and Ludger Wößmann, Does Educational Tracking Affect Performance and Inequality? Differences-in-Differences Evidence across Countries, February 2005

1416 George Kapetanios and M. Hashem Pesaran, Alternative Approaches to Estimation and Inference in Large Multifactor Panels: Small Sample Results with an Application to Modelling of Asset Returns, February 2005

1417 Samuel Mühlemann, Jürg Schweri, Rainer Winkelmann and Stefan C. Wolter, A Structural Model of Demand for Apprentices. February 2005

1418 Giorgio Brunello and Lorenzo Rocco, Educational Standards in Private and Public Schools, February 2005

1419 Alex Bryson, Lorenzo Cappellari and Claudio Lucifora, Why so Unhappy? The Effects of Unionisation on Job Satisfaction, March 2005

1420 Annalisa Luporini, Relative Performance Evaluation in a Multi-Plant Firm, March 2005

1421 Giorgio Bellettini and Carlotta Berti Ceroni, When the Union Hurts the Workers: A Positive Analysis of Immigration Policy, March 2005

1422 Pieter Gautier, Michael Svarer and Coen Teulings, Marriage and the City, March 2005

1423 Ingrid Ott and Stephen J. Turnovsky, Excludable and Non-Excludable Public Inputs: Consequences for Economic Growth, March 2005

1424 Frederick van der Ploeg, Back to Keynes?, March 2005 
1425 Stephane Dees, Filippo di Mauro, M. Hashem Pesaran and L. Vanessa Smith, Exploring the International Linkages of the Euro Area: a Global VAR Analysis, March 2005

1426 Hans Pitlik, Friedrich Schneider and Harald Strotmann, Legislative Malapportionment and the Politicization of Germany’s Intergovernmental Transfer System, March 2005

1427 Konstantinos Angelopoulos and Apostolis Philippopoulos, The Role of Government in Anti-Social Redistributive Activities, March 2005

1428 Ansgar Belke and Daniel Gros, Asymmetries in the Trans-Atlantic Monetary Policy Relationship: Does the ECB follow the Fed?, March 2005

1429 Sören Blomquist and Luca Micheletto, Optimal Redistributive Taxation when Government’s and Agents’ Preferences Differ, March 2005

1430 Olof Åslund and Peter Fredriksson, Ethnic Enclaves and Welfare Cultures - QuasiExperimental Evidence, March 2005

1431 Paul De Grauwe, Roberto Dieci and Marianna Grimaldi, Fundamental and NonFundamental Equilibria in the Foreign Exchange Market. A Behavioural Finance Framework, March 2005

1432 Peter Egger, Stefan Gruber, Mario Larch and Michael Pfaffermayr, Knowledge-Capital Meets New Economic Geography, March 2005

1433 George Economides and Apostolis Philippopoulos, Should Green Governments Give Priority to Environmental Policies over Growth-Enhancing Policies?, March 2005

1434 George W. Evans and Seppo Honkapohja, An Interview with Thomas J. Sargent, March 2005

1435 Helge Berger and Volker Nitsch, Zooming Out: The Trade Effect of the Euro in Historical Perspective, March 2005

1436 Marc-Andreas Muendler, Rational Information Choice in Financial Market Equilibrium, March 2005

1437 Martin Kolmar and Volker Meier, Intra-Generational Externalities and InterGenerational Transfers, March 2005

1438 M. Hashem Pesaran and Takashi Yamagata, Testing Slope Homogeneity in Large Panels, March 2005

1439 Gjermund Nese and Odd Rune Straume, Industry Concentration and Strategic Trade Policy in Successive Oligopoly, April 2005

1440 Tomer Blumkin and Efraim Sadka, A Case for Taxing Education, April 2005

1441 John Whalley, Globalization and Values, April 2005 
1442 Denise L. Mauzerall, Babar Sultan, Namsoug Kim and David F. Bradford, Charging $\mathrm{NO}_{x}$ Emitters for Health Damages: An Exploratory Analysis, April 2005

1443 Britta Hamburg, Mathias Hoffmann and Joachim Keller, Consumption, Wealth and Business Cycles in Germany, April 2005

1444 Kohei Daido and Hideshi Itoh, The Pygmalion Effect: An Agency Model with Reference Dependent Preferences, April 2005

1445 John Whalley, Rationality, Irrationality and Economic Cognition, April 2005

1446 Henning Bohn, The Sustainability of Fiscal Policy in the United States, April 2005

1447 Torben M. Andersen, Is there a Role for an Active Fiscal Stabilization Policy? April 2005

1448 Hans Gersbach and Hans Haller, Bargaining Power and Equilibrium Consumption, April 2005

1449 Jerome L. Stein, The Transition Economies: A NATREX Evaluation of Research, April 2005

1450 Raymond Riezman, John Whalley and Shunming Zhang, Metrics Capturing the Degree to which Individual Economies are Globalized, April 2005

1451 Romain Ranciere, Aaron Tornell and Frank Westermann, Systemic Crises and Growth, April 2005

1452 Plutarchos Sakellaris and Focco W. Vijselaar, Capital Quality Improvement and the Sources of Growth in the Euro Area, April 2005

1453 Kevin Milligan and Michael Smart, Regional Grants as Pork Barrel Politics, April 2005

1454 Panu Poutvaara and Andreas Wagener, To Draft or not to Draft? Efficiency, Generational Incidence, and Political Economy of Military Conscription, April 2005

1455 Maurice Kugler and Hillel Rapoport, Skilled Emigration, Business Networks and Foreign Direct Investment, April 2005

1456 Yin-Wong Cheung and Eiji Fujii, Cross-Country Relative Price Volatility: Effects of Market Structure, April 2005

1457 Margarita Katsimi and Thomas Moutos, Inequality and Relative Reliance on Tariffs: Theory and Evidence, April 2005

1458 Monika Bütler, Olivia Huguenin and Federica Teppa, Why Forcing People to Save for Retirement may Backfire, April 2005

1459 Jos Jansen, The Effects of Disclosure Regulation of an Innovative Firm, April 2005 
1460 Helge Bennmarker, Kenneth Carling and Bertil Holmlund, Do Benefit Hikes Damage Job Finding? Evidence from Swedish Unemployment Insurance Reforms, May 2005

1461 Steffen Huck, Kai A. Konrad and Wieland Müller, Merger without Cost Advantages, May 2005

1462 Louis Eeckhoudt and Harris Schlesinger, Putting Risk in its Proper Place, May 2005

1463 Hui Huang, John Whalley and Shunming Zhang, Trade Liberalization in a Joint Spatial Inter-Temporal Trade Model, May 2005

1464 Mikael Priks, Optimal Rent Extraction in Pre-Industrial England and France - Default Risk and Monitoring Costs, May 2005

1465 François Ortalo-Magné and Sven Rady, Heterogeneity within Communities: A Stochastic Model with Tenure Choice, May 2005

1466 Jukka Pirttilä and Sanna Tenhunen, Pawns and Queens Revisited: Public Provision of Private Goods when Individuals make Mistakes, May 2005

1467 Ernst Fehr, Susanne Kremhelmer and Klaus M. Schmidt, Fairness and the Optimal Allocation of Ownership Rights, May 2005

1468 Bruno S. Frey, Knight Fever - Towards an Economics of Awards, May 2005

1469 Torberg Falch and Marte Rønning, The Influence of Student Achievement on Teacher Turnover, May 2005

1470 John Komlos and Peter Salamon, The Poverty of Growth with Interdependent Utility Functions, May 2005

1471 Hui Huang, Yi Wang, Yiming Wang, John Whalley and Shunming Zhang, A Trade Model with an Optimal Exchange Rate Motivated by Current Discussion of a Chinese Renminbi Float, May 2005

1472 Helge Holden, Lars Holden and Steinar Holden, Contract Adjustment under Uncertainty, May 2005 